TRANSACTIONS OF THE

AMERICAN MATHEMATICAL SOCIETY

Volume 362, Number 6, June 2010, Pages 2875-2899

S 0002-9947(10)04733-1

Article electronically published on January 4, 2010

\title{
ON A QUESTION OF LANDIS AND OLEINIK
}

\author{
TU A. NGUYEN
}

\begin{abstract}
Let $P=\partial_{t}+\partial_{i}\left(a^{i j} \partial_{j}\right)$ be a backward parabolic operator. It is shown that under certain conditions on $\left\{a^{i j}\right\}$, if $u$ satisfies $|P u| \leq$ $C(|u|+|\nabla u|),|u(x, t)| \lesssim e^{C|x|^{2}}$ in $\mathbb{R}^{n} \times[0, T]$ and $|u(x, 0)| \lesssim e^{-M|x|^{2}}$ for all $M>0$, then $u$ vanishes identically in $\mathbb{R}^{n} \times[0, T]$.
\end{abstract}

\section{INTRODUCTION}

Let $P$ be a backward parabolic operator on $\mathbb{R}^{n}$,

$$
P u=\partial_{t} u+\operatorname{div}(A \nabla u),
$$

where $A(x, t)=\left(a^{i j}(x, t)\right)_{i, j=1}^{n}$ is a real, symmetric matrix such that for some $\lambda>0$,

$$
\lambda|\xi|^{2} \leq a^{i j}(x, t) \xi_{i} \xi_{j} \leq \lambda^{-1}|\xi|^{2} \text { for all } \xi \in \mathbb{R}^{n} .
$$

It was conjectured by Landis and Oleinik [1] that if $P u=b(x, t) \cdot \nabla u+a(x, t) u$ in $\mathbb{R}^{n} \times[0, T]$ and $|u(x, 0)| \lesssim e^{-|x|^{2+\epsilon}}, \forall x \in \mathbb{R}^{n}$, then $u \equiv 0$ in $\mathbb{R}^{n} \times[0, T]$, provided $A, b$ and $c$ satisfy the appropriate conditions at infinity.

Escauriaza, Kenig, Ponce and Vega 3 showed that this is true when $P$ is the backward heat operator (i.e. $A(x, t) \equiv \mathrm{Id}$ ) and $b$ and $c$ are bounded. They also obtained a similar result when the domain is $\mathbb{R}_{+}^{n} \times[0, T]$. The aim of this paper is to extend these results to parabolic operators with variable coefficients. Our main result is the following.

Theorem 1.1. Suppose that $\left\{a^{i j}\right\}$ satisfy the ellipticity condition (1), and for some $\epsilon>0$,

$$
\begin{aligned}
& \left|\nabla_{x} a^{i j}(x, t)\right| \lesssim\langle x\rangle^{-1-\epsilon},\left|\partial_{t} a^{i j}(x, t)\right| \lesssim 1, \\
& \left|a^{i j}(x, t)-a^{i j}(x, s)\right| \lesssim\langle x\rangle^{-1}|t-s|^{1 / 2}, \quad \forall x \in \mathbb{R}^{n} ; t, s \in[0, T] .
\end{aligned}
$$

Assume that $u$ satisfies the inequalities

$$
|P u| \leq C(|u|+|\nabla u|) \quad \text { in } \mathbb{R}^{n} \times[0, T]
$$

and

$$
|u(x, t)| \lesssim e^{C|x|^{2}} \quad \forall(x, t) \in \mathbb{R}^{n} \times[0, T],
$$

for some $C>0$. Then

1. If $|u(x, 0)| \lesssim e^{-M|x|^{2}}$ for all $M>0$, then $u \equiv 0$.

Received by the editors October 29, 2007.

2000 Mathematics Subject Classification. Primary 35K99.

(C)2010 American Mathematical Society Reverts to public domain 28 years from publication 
2. If $u(x, 0) \not \equiv 0$, then there exists $M>0$ such that if $|x|>M$,

$$
\int_{B(x, 1)}|u(y, 0)|^{2} d y \gtrsim e^{-M|x|^{2} \log |x|} \quad \text { and } \quad \int_{B(x,|x| / 2)}|u(y, 0)|^{2} d y \gtrsim e^{-M|x|^{2}} .
$$

We also obtain a similar result where the domain is a half-space.

Theorem 1.2. Let $\mathbb{R}_{+}^{n}=\left\{x \in \mathbb{R}^{n}: x_{1}>0\right\}$. Suppose that $\left\{a^{i j}\right\}$ satisfy the ellipticity condition (11), and for some $\epsilon>0$,

$$
\begin{aligned}
& \left|\nabla_{x} a^{i j}(x, t)\right| \lesssim\langle x\rangle^{-1-\epsilon},\left|\partial_{t} a^{i j}(x, t)\right| \lesssim 1, \\
& \left|a^{i j}(x, t)-a^{i j}(x, s)\right| \lesssim\langle x\rangle^{-1}|t-s|^{1 / 2}, \quad \forall x \in \mathbb{R}_{+}^{n} ; \quad t, s \in[0, T] .
\end{aligned}
$$

Assume that $u$ satisfies the inequalities

$$
|P u| \leq C(|u|+|\nabla u|) \quad \text { in } \mathbb{R}_{+}^{n} \times[0, T]
$$

and

$$
|u(x, t)| \lesssim e^{C|x|^{2}} \quad \forall(x, t) \in \mathbb{R}_{+}^{n} \times[0, T],
$$

for some $C>0$. Then

1. If $|u(x, 0)| \lesssim e^{C|x|^{2}-M x_{1}^{2}}$ for all $M>0$, then $u \equiv 0$.

2. If $u(\cdot, 0) \not \equiv 0$, then there exists $M>0$ such that if $R>M$,

$$
\int_{B\left(R e_{1}, 1\right)}|u(y, 0)|^{2} d y \gtrsim e^{-M R^{2} \log R} \quad \text { and } \quad \int_{B\left(R e_{1}, R / 2\right)}|u(y, 0)|^{2} d y \gtrsim e^{-M R^{2}} .
$$

The proof in [3] for the case $A(x, t) \equiv \mathrm{Id}$ uses a Carleman inequality together with a scaling argument to show that $u(x, 0)$ has a doubling property which implies the following lower bound:

$$
\int_{B(x,|x| / 2)}|u(y, 0)|^{2} d y \gtrsim e^{-M|x|^{2}}
$$

for some $M>0$. This argument requires a uniform bound on $\left\|\nabla a_{x_{0}, R}^{i j}\right\|_{L^{\infty}}$, where $a_{x_{0}, R}^{i j}(x, t)=a^{i j}\left(x_{0}+R x, R^{2} t\right)$ and $R>\left|x_{0}\right|$; hence it does not apply to the variable coefficients case.

To prove the first part of Theorem 1.1, we first show that if $|u(x, 0)| \lesssim e^{-M|x|^{2}}$ for all $M>0$, then there exists $T_{0} \in(0, T]$ such that for any $M \geq 0$,

$$
\int_{0}^{T_{0}} \int_{B(x,|x| / 2)} u^{2}(y, t) d y d t \lesssim e^{-M|x|^{2}} \quad \text { if }|x| \geq R_{M} .
$$

Then we show that if $u(\cdot, 0) \not \equiv 0$, for any $T_{0} \in(0, T]$, the following lower bound holds:

$$
\int_{0}^{T_{0}} \int_{B(x,|x| / 2)} u^{2}(y, t) d y d t \gtrsim e^{-C_{2}|x|^{2}}
$$

where $C_{2}=C_{2}\left(T_{0}, u\right) \geq 0$. (A similar bound for the Schrödinger equation was proved in [4.) Thus, we must have $u(\cdot, 0) \equiv 0$, which then implies $u \equiv 0$ in $\mathbb{R}^{n} \times[0, T]$. The proof of the second part of Theorem 1.1 is a slight modification of the proof of the first part. The proof of Theorem 1.2 follows the same lines, using anisotropic Carleman inequalities instead, as now $u$ decays in the direction of $x_{1}$ only.

We would like to mention a unique continuation result of [7, 8] and [1]. Let $u$ be a solution of the inequality $|P u| \leq M(|u|+|\nabla u|)$ in $B(0,1) \times[0, T]$ which vanishes 
to infinite order at 0 , i.e. $|u(x, 0)| \leq C_{k}|x|^{k}$ for all $k \geq 0, \forall x \in B(0,1)$. Then $u(\cdot, 0) \equiv 0$ in $B(0,1)$. We have benefited from the Carleman inequalities and ideas contained in [7, 8, and also from those of [9, 6, 5, 4, 3,

The details of the proofs of Theorems 1.1 and 1.2 are in sections 2 and 3, respectively. The proofs of the Carleman inequalities used in sections 2 and 3 will be gathered in section 4 , together with other auxiliary lemmas.

\section{Proof of Theorem 1.1}

We first remark that by considering $P_{r}=\partial_{t}+\operatorname{div}\left(A_{r} \nabla\right)$, where $a_{r}^{i j}(x, t)=$ $a^{i j}\left(r x, r^{2} t\right)$ and $u_{r}(x, t)=u\left(r x, r^{2} t\right)$ for suitably small $r>0$, we can assume that the constant $C$ in the hypothesis of Theorem 1.1 is as small as we like, say $C \leq \lambda^{5} / 100$, and that $\left|\partial_{t} a^{i j}(x, t)\right| \leq C$. Furthermore, without loss of generality, we can assume $T=1$.

2.1. Upper bound. In this section we will adapt the arguments of [5] to show that under the hypothesis of Theorem 1.1 , there exists $T \in(0,1]$ such that for any $M>0$, if $|x| \geq R_{M}>0$,

$$
|u(x, t)|+|\nabla u(x, t)| \lesssim e^{-M|x|^{2}} \text { for all } t \in[0, T] .
$$

First, we prove the weaker bound

$$
|u(x, t)|+|\nabla u(x, t)| \lesssim e^{-M|x|^{2}} \quad \text { if } 0 \leq t \lesssim M^{-1} .
$$

(Note that the time interval of this weaker bound shrinks as $M \rightarrow \infty$.) Then we combine this bound with $M=2$ and another Carleman inequality to obtain (2).

2.1.1. First step. We will use the following Carleman inequality of [7].

Lemma 2.1. Suppose $a^{i j}(0,0)=\delta_{i j}$ and $\left|a^{i j}(x, t)-a^{i j}(y, s)\right| \leq L\left(|x-y|+|s-t|^{1 / 2}\right)$. Then there is a constant $N=N(n, \lambda, L)>0$ such that for any $\alpha \geq 2$ there is a positive function $\sigma:\left(0, \frac{4}{\alpha}\right) \rightarrow \mathbb{R}_{+}$satisfying

$$
N^{-1} \leq \frac{\sigma(t)}{t} \leq 1
$$

so that if $v \in C_{c}^{\infty}\left(\mathbb{R}^{n} \times\left[0, \frac{2}{\alpha}\right)\right)$ and $0<a<1 / \alpha$, then

$$
\begin{gathered}
\int_{\mathbb{R}^{n+1}}\left(\alpha^{2} v^{2}+\alpha \sigma_{a}|\nabla v|^{2}\right) \sigma_{a}^{-\alpha} G_{a} d x d t \leq N \int_{\mathbb{R}^{n+1}} \sigma_{a}^{1-\alpha}|P v|^{2} G_{a} d x d t \\
+\sigma(a)^{-\alpha}\left[-\frac{a}{N} \int_{\mathbb{R}^{n}}|\nabla v(x, 0)|^{2} G_{a}(x, 0) d x+\alpha N \int_{\mathbb{R}^{n}} v^{2}(x, 0) G_{a}(x, 0) d x\right] \\
+\alpha^{\alpha} N^{\alpha} \sup _{t \geq 0} \int_{\mathbb{R}^{n}}\left(v^{2}+|\nabla v|^{2}\right) d x . \\
\text { Here } G_{a}(x, t)=(t+a)^{-n / 2} e^{-|x|^{2} / 4(t+a)} \text { and } \sigma_{a}(t)=\sigma(t+a) .
\end{gathered}
$$

Since the hypothesis of the lemma requires $a^{i j}(0,0)=\delta_{i j}$, we first need to make a change of variable. Let $x_{0} \in \mathbb{R}^{n}$ with $\left|x_{0}\right| \gtrsim 1$. Let $S=A\left(x_{0}, 0\right)^{1 / 2}, z_{0}=S^{-1} x_{0}$, $\widetilde{u}(x, t)=u(S x, t)$ and $\widetilde{A}(x, t)=S^{-1} A(S x, t) S^{-1}$. Then $\widetilde{A}\left(z_{0}, 0\right)=\mathrm{Id}$ and

$$
\partial_{t} \widetilde{u}+\left.\operatorname{div}(\widetilde{A} \nabla \widetilde{u})\right|_{(x, t)}=\partial_{t} u+\left.\operatorname{div}(A \nabla u)\right|_{(S x, t)} .
$$


Let $\widetilde{u}_{R}$ be a rescaling of $\widetilde{u}$ centered at $z_{0}, \widetilde{u}_{R}(x, t)=\widetilde{u}\left(z_{0}+R x, R^{2} t\right)$, where $R=$ $\lambda\left|x_{0}\right| / 4$. Then $\widetilde{u}_{R}$ satisfies

$$
\left|P_{R} \widetilde{u}_{R}\right| \leq R^{2}\left|\widetilde{u}_{R}\right|+R\left|\nabla \widetilde{u}_{R}\right|,
$$

where $P_{R}=\partial_{t}+\operatorname{div}\left(\widetilde{A}_{R} \nabla\right)$ and $\widetilde{A}_{R}(x, t)=\widetilde{A}\left(z_{0}+R x, R^{2} t\right)$.

From the hypothesis of Theorem 1.1 and our choice of $R$, it is easy to see that

$$
\left|\nabla \widetilde{a}_{R}^{i j}(x, t)\right| \lesssim 1,\left|\widetilde{a}_{R}^{i j}(x, t)-\widetilde{a}_{R}^{i j}(x, s)\right| \lesssim|t-s|^{1 / 2}
$$

in $B(0,2) \times\left[0,1 / R^{2}\right)$. Furthermore, $\widetilde{A}_{R}(0,0)=\widetilde{A}\left(z_{0}, 0\right)=$ Id. Thus, we can apply Lemma 2.1 to $P_{R}$ and $v=\widetilde{u}_{R} \psi(x) \varphi(t)$, where $\chi_{[0,1 / \alpha]} \leq \varphi \leq \chi_{[0,2 / \alpha)}$ and $\chi_{B(0,1)} \leq \psi \leq \chi_{B(0,2)}$ are bump functions, and $\alpha \geq 2 R^{2}$ is a positive constant to be chosen. Let $E=B(0,2) \times[0,2 / \alpha) \backslash B(0,1) \times[0,1 / \alpha]$. Then

$$
\left|P_{R} v\right| \leq R^{2}|v|+R|\nabla v|+\alpha\left(\left|\widetilde{u}_{R}\right|+\left|\nabla \widetilde{u}_{R}\right|\right) \chi_{E} .
$$

Hence, by Lemma 2.1

$$
\begin{aligned}
\int_{\mathbb{R}^{n+1}}\left(\alpha^{2} v^{2}+\alpha \sigma_{a}|\nabla v|^{2}\right) \sigma_{a}^{-\alpha} G_{a} d x d t \lesssim & N \int_{\mathbb{R}^{n+1}} \sigma_{a}^{1-\alpha}\left(R^{2}|v|+R|\nabla v|\right)^{2} G_{a} d x d t \\
& +N \int_{E} \sigma_{a}^{1-\alpha} \alpha^{2}\left(\left|\widetilde{u}_{R}\right|+\left|\nabla \widetilde{u}_{R}\right|\right)^{2} G_{a} d x d t \\
& +\alpha^{\alpha} N^{\alpha} \sup _{t \geq 0} \int_{\mathbb{R}^{n}}\left(v^{2}+|\nabla v|^{2}\right) d x \\
& +\alpha N \sigma(a)^{-\alpha} \int_{\mathbb{R}^{n}} v^{2}(x, 0) G_{a} d x .
\end{aligned}
$$

If $\alpha \geq 2 N R^{2}$, then the first term on the right hand side can be absorbed by the left hand side. In $E, \sigma_{a}(t)^{-\alpha} G_{a}(x, t) \leq \alpha^{\alpha+\frac{n}{2}} N^{\alpha}$, and $\left|\widetilde{u}_{R}\right|+\left|\nabla \widetilde{u}_{R}\right| \lesssim e^{C R^{2}}$ by hypothesis on $u$ and Lemma 4.1 in the Appendix. Hence, we obtain

$$
\begin{aligned}
\int_{\mathbb{R}^{n+1}}\left(\alpha^{2} v^{2}+\alpha \sigma_{a}|\nabla v|^{2}\right) \sigma_{a}^{-\alpha} G_{a} d x d t \lesssim & \alpha^{\alpha+\frac{n}{2}} e^{2 C R^{2}} N^{\alpha} \\
& +\alpha N \sigma(a)^{-\alpha} \int_{\mathbb{R}^{n}} v^{2}(x, 0) G_{a} d x .
\end{aligned}
$$

Let $\rho=\frac{1}{N e}$, and $a=\frac{\rho^{2}}{2 \alpha}$. Then

$$
\sigma_{a}(t)^{-\alpha+1} G_{a}(x, t) \geq \alpha^{\alpha+\frac{n}{2}-1} N^{2 \alpha+n-2} \quad \text { in } B(0,2 \rho) \times\left[0, \frac{\rho^{2}}{2 \alpha}\right]
$$

and

$$
\sigma(a)^{-\alpha} G_{a}(x, 0) \leq a^{-\alpha-\frac{n}{2}} N^{\alpha}=\left(2 \alpha e^{2}\right)^{\alpha+\frac{n}{2}} N^{3 \alpha+n} \text { for all } x .
$$

Therefore, we deduce from (4) that

or

$$
\begin{gathered}
\alpha^{\alpha+\frac{n}{2}} N^{2 \alpha+n-2} \int_{B(0,2 \rho) \times\left[0, \frac{\rho^{2}}{2 \alpha}\right]}\left(v^{2}+|\nabla v|^{2}\right) d x d t \lesssim \alpha^{\alpha+\frac{n}{2}} e^{2 C R^{2}} N^{\alpha} \\
+\alpha^{\alpha+\frac{n}{2}+1}(2 e)^{2 \alpha+n} N^{3 \alpha+n+1} \int_{B(0,2)} v^{2}(x, 0) d x
\end{gathered}
$$

$\int_{B(0,2 \rho) \times\left[0, \frac{\rho^{2}}{2 \alpha}\right]}\left(v^{2}+|\nabla v|^{2}\right) d x d t \lesssim e^{2 C R^{2}} N^{2-\alpha-n}+\alpha(2 e)^{2 \alpha+n} N^{\alpha+3} \int_{B(0,2)} v^{2}(x, 0) d x$. 
We now choose $\alpha=M R^{2}$. Then the first term on the right hand side is bounded by $e^{-M R^{2}}$. The second term is also bounded by $e^{-M R^{2}}$ by the decay hypothesis on $u(\cdot, 0)$. Thus, for any $M>2 N$,

$$
\int_{B(0,2 \rho) \times\left[0, \frac{\rho^{2}}{2 M R^{2}}\right]}\left(v^{2}+|\nabla v|^{2}\right) d x d t \lesssim e^{-M R^{2}} .
$$

By Lemma 4.1, this implies

$$
|v|+|\nabla v| \lesssim e^{-M R^{2}} \quad \text { in } B(0, \rho) \times\left[0, \frac{\rho^{2}}{4 M R^{2}}\right] .
$$

Undoing the change of variable, we get

$$
\left|u\left(x_{0}, t\right)\right|+\left|\nabla u\left(x_{0}, t\right)\right| \lesssim e^{-M R^{2}} \quad \text { if } 0 \leq t \leq \frac{\rho^{2}}{4 M} .
$$

This proves (3).

\subsubsection{Second step.}

Lemma 2.2. Let $\epsilon$ be the constant in the hypothesis of Theorem 1.1 and

$$
G(x, t)=\exp \left(c(T-t)|x|+|x|^{2}\right),
$$

where $0 \leq c \leq R^{1+\epsilon / 8}$. Then for any $v \in C_{c}^{\infty}\left(\left\{R \leq|x| \leq R^{1+\epsilon / 8}\right\} \times[0, T]\right)$, the following inequality holds:

$$
\begin{aligned}
& \frac{\lambda^{2}}{4} \int_{\mathbb{R}^{n+1}} v^{2} G d x d t+\frac{\lambda^{2}}{4} \int_{\mathbb{R}^{n+1}}|\nabla v|^{2} G d x d t \leq \int_{\mathbb{R}^{n+1}}|P v|^{2} G d x d t \\
& +\lambda^{-1} \int_{\mathbb{R}^{n}}|\nabla v(x, T)|^{2} G(x, T) d x+R^{2+\epsilon / 4} \int_{\mathbb{R}^{n}} v^{2}(x, 0) G(x, 0) d x,
\end{aligned}
$$

provided $R \gtrsim 1$.

This Carleman inequality is an extension of a Carleman inequality in [5] to the case of variable coefficients. As $\left\{a^{i j}\right\}$ are no longer constants, it is necessary to put a restriction on the support of $v$ (compared with inequality (1.5) of [5]). We will prove this inequality in the Appendix. We now deduce (2) from (3) and Lemma 2.2 .

Proposition 2.3. Suppose that $u$ is as in the hypothesis of Theorem 1.1, and $|u(x, 0)| \lesssim e^{-M|x|^{2}}$ for all $M>0$. Let $T=\rho^{2} /(8 N)$, where $\rho$ and $N$ are as above. Then for all $M>0$,

$$
|u(x, t)|+|\nabla u(x, t)| \lesssim e^{-M|x|^{2}}
$$

for all $t \in[0, T / 4]$.

Proof. Fix $M>0$. Let

$$
v(x, t)=u(x, t) \theta(x),
$$

where

$$
\theta(x)=\left\{\begin{array}{cc}
0 & \text { if }|x|<R-1 \text { or }|x|>M R+1, \\
1 & \text { if } R<|x|<M R .
\end{array}\right.
$$

Since

$$
P v=\theta P u+2\langle A \nabla u, \nabla \theta\rangle+u \Delta \theta
$$


it follows that

$$
\begin{aligned}
|P v| & \leq C \theta(|u|+|\nabla u|)+2 \lambda^{-1}|\nabla u||\nabla \theta|+|u \Delta \theta| \\
& \leq C(|v|+|\nabla v|)+|u|(C|\nabla \theta|+|\Delta \theta|)+2 \lambda^{-1}|\nabla u||\nabla \theta| \\
& \leq C(|v|+|\nabla v|)+C^{\prime}(|u|+|\nabla u|) \chi_{E},
\end{aligned}
$$

where $E=(\{R-1<|x|<R\} \cup\{M R<|x|<M R+1\}) \times[0, T], C^{\prime} \leq 4 \lambda^{-1}$.

Choose $c=M R / T$. Then for large $R, c \leq R^{1+\epsilon / 8}$ and $\operatorname{supp} v \subset\{R \leq|x|$ $\left.\leq R^{1+\epsilon / 8}\right\}$. Thus, we can apply the previous lemma to $v$ to obtain

$$
\begin{aligned}
\int_{0}^{T} \int_{\mathbb{R}^{n}}\left(v^{2}+|\nabla v|^{2}\right) G d x d t \lesssim & \int_{\mathbb{R}^{n}}|\nabla v(x, T)|^{2} G(x, T) d x \\
& +R^{2+\epsilon / 4} \int_{\mathbb{R}^{n}} v^{2}(x, 0) G(x, 0) d x \\
& +\int_{E}\left(|u|^{2}+|\nabla u|^{2}\right) G d x d t .
\end{aligned}
$$

In the previous subsection, we have shown that

$$
|u(x, t)|+|\nabla u(x, t)| \lesssim e^{-2 N|x|^{2}} \text { for all } t \in[0, T] .
$$

Hence, as $G(x, T)=e^{|x|^{2}}$, we have

$$
\int_{\mathbb{R}^{n}}|\nabla v(x, T)|^{2} G d x \lesssim 1
$$

Since $|u(x, 0)| \lesssim e^{-2 M|x|^{2}}$ and $G(x, 0) \leq e^{(M+1)|x|^{2}}$ if $|x| \geq R$ by our choice of $c$, it follows that

$$
R^{2+\epsilon / 4} \int_{\mathbb{R}^{n}} v^{2}(x, 0) G d x \lesssim 1
$$

In $\{M R<|x|<M R+1\}, G(x, t) \leq e^{2|x|^{2}}$; hence,

$$
\int_{0}^{T} \int_{M R<|x|<M R+1}\left(|u|^{2}+|\nabla u|^{2}\right) G d x d t \lesssim R^{n-1} .
$$

In $\{R-1<|x|<R\}, G(x, t) \leq e^{(M+1) R^{2}}$, so

$$
\int_{0}^{T} \int_{R-1<|x|<R}\left(|u|^{2}+|\nabla u|^{2}\right) G d x d t \lesssim e^{(M+2) R^{2}} .
$$

Thus,

$$
\int_{0}^{T} \int_{\mathbb{R}^{n}}\left(v^{2}+|\nabla v|^{2}\right) G d x d t \lesssim e^{(M+2) R^{2}} .
$$

As $G(x, t) \geq e^{4 M R^{2}}$ in $\{6 R \leq|x| \leq 7 R\} \times[0, T / 2]$, this implies

$$
\int_{0}^{T / 2} \int_{6 R \leq|x| \leq 7 R}\left(|u|^{2}+|\nabla u|^{2}\right) d x d t \lesssim e^{-M R^{2}}
$$

provided $R \geq R_{M}$. The proposition follows from this and Lemma 4.1 
2.2. Lower bound. In this subsection, assuming $u(\cdot, 0) \not \equiv 0$, we will show that the following lower bound holds for any $T \leq 1$ :

$$
\int_{0}^{T} \int_{R<|x|<2 R} u^{2}(x, 0) d x \gtrsim e^{-C_{2} R^{2}}
$$

To prove this, we first adapt arguments of [2] and [9] to show that there exists $s>0$, such that for small $t$, we have

$$
\int_{R<|x|<2 R} u^{2}(x, t) d x \gtrsim e^{-R^{s}} .
$$

Then we use this bound together with a bootstrap argument to obtain (5).

2.2.1. First step. Since $u(\cdot, 0) \not \equiv 0$, we can suppose that

$$
\int_{B\left(e_{1}, \rho \lambda / 4\right)} u^{2}(x, 0) d x \neq 0
$$

Here $\rho$ is a positive constant to be chosen. By using Lemma 4.2, and multiplying $u$ by a constant if necessary, we can assume that

$$
\int_{B\left(e_{1}, \rho \lambda / 2\right)} u^{2}(x, t) d x \geq L
$$

if $t$ is small enough. Here $L$ is a large constant to be chosen.

We will use the doubling property of $u(\cdot, 0)$ proved by Escauriaza, Fernández and Vessella [2]. We present their arguments here in the form that we need. Let $x_{0}=\left|x_{0}\right| e_{1}$, and $v$ be as in section 2.1. As before, if $\alpha \geq 2 N R^{2}$, the following inequality holds:

$$
\begin{aligned}
& \int_{\mathbb{R}^{n+1}}\left(\alpha^{2} v^{2}+\alpha \sigma_{a}|\nabla v|^{2}\right) \sigma_{a}^{-\alpha} G_{a} d x d t \leq N^{\alpha} \alpha^{\alpha+\frac{n}{2}} e^{C R^{2}} \\
& +\sigma(a)^{-\alpha}\left[-\frac{a}{N} \int_{\mathbb{R}^{n}}|\nabla v(x, 0)|^{2} G_{a} d x+\alpha N \int_{\mathbb{R}^{n}} v^{2}(x, 0) G_{a} d x\right] .
\end{aligned}
$$

Let $\rho=\frac{1}{N e}$ and $0<a \leq \rho^{2} /(2 \alpha)$. Then,

$$
\begin{aligned}
\alpha^{2} \int_{\mathbb{R}^{n+1}} v^{2} \sigma_{a}^{-\alpha} G_{a} & \geq \alpha^{2} \int_{0}^{\rho^{2} / \alpha} d t \int_{B(0,2 \rho)}(t+a)^{-\alpha-\frac{n}{2}} e^{-\rho^{2} /(t+a)} v^{2}(x, t) d x \\
& \geq N_{\rho} \alpha^{2} \int_{a}^{a+\rho^{2} / \alpha} s^{-\alpha-\frac{n}{2}} e^{-\rho^{2} / s} d s \int_{B(0, \rho)} v^{2}(x, 0) d x \\
& \geq N_{\rho} \alpha^{2} \int_{\rho^{2} / 2 \alpha}^{\rho^{2} / \alpha} s^{-\alpha-\frac{n}{2}} e^{-\rho^{2} / s} d s \int_{B(0, \rho)} v^{2}(x, 0) d x \\
& \geq \frac{N_{\rho} \alpha^{\alpha+\frac{n}{2}+1} N^{2 \alpha}}{2} \int_{B(0, \rho)} v^{2}(x, 0) d x
\end{aligned}
$$

(We have used Lemma 4.2 in the second inequality. $N_{\rho}$ is the constant appearing in that lemma.) Here, $\alpha$ has to satisfy

$$
\rho^{2} / \alpha \leq N_{\rho}^{-1} \min \left\{R^{-2}, 1 / \log \left(\frac{N_{\rho} \int_{B(0,1) \times\left[0, R^{-2}\right]} v^{2}(x, t) d x d t}{\int_{B(0, \rho)} v^{2}(x, 0) d x}\right)\right\} .
$$


As $|v(x, t)| \lesssim e^{C R^{2}}$, we can take

$$
\alpha=\rho^{2} N_{\rho}\left(2 R^{2}+\log \frac{N_{\rho}}{\int_{B(0, \rho)} v^{2}(x, 0) d x}\right) .
$$

For this value of $\alpha$,

$$
\frac{N_{\rho} \alpha^{\alpha+\frac{n}{2}+1} N^{2 \alpha}}{2} \int_{B(0, \rho)} v^{2}(x, 0) d x \geq N^{\alpha} \alpha^{\alpha+\frac{n}{2}} e^{C R^{2}} .
$$

This together with (8) and (9) shows that

$$
-\frac{a}{N} \int_{\mathbb{R}^{n}}|\nabla v(x, 0)|^{2} G_{a}(x, 0) d x+\alpha N \int_{\mathbb{R}^{n}} v^{2}(x, 0) G_{a}(x, 0) d x \geq 0
$$

or

$$
\begin{aligned}
& 2 a \int_{\mathbb{R}^{n}}|\nabla v(x, 0)|^{2} G_{a}(x, 0) d x+\frac{n}{2} \int_{\mathbb{R}^{n}} v^{2}(x, 0) G_{a}(x, 0) d x \\
& \quad \leq 4 \alpha N^{2} \int_{\mathbb{R}^{n}} v^{2}(x, 0) G_{a}(x, 0) d x
\end{aligned}
$$

for all $a \leq \rho^{2} /(2 \alpha)$.

By Lemma 4.3, this implies that

$$
\int_{B(0,2 r)} v^{2}(x, 0) \leq e^{128 \alpha N^{2}} \int_{B(0, r)} v^{2}(x, 0)
$$

for all $0 \leq r \leq 1 / 2$. It follows that there exist positive constant $C_{1}$ and $C_{2}$ such that if $r \leq \rho / 2$,

$$
\left(\int_{B(0, \rho)} v^{2}(x, 0)\right)^{1+C_{1} \log \frac{\rho}{r}} \leq e^{C_{2} R^{2} \log \frac{\rho}{r}} \int_{B(0, r)} v^{2}(x, 0) .
$$

Taking $r=\rho \lambda^{2} / 2$, we see that there are constants $J$ and $K$ so that

$$
\left(\int_{B(0, \rho)} v^{2}(x, 0)\right)^{K} \leq e^{J R^{2} / 2} \int_{B\left(0, \rho \lambda^{2} / 2\right)} v^{2}(x, 0) .
$$

This implies, after undoing the changes of variable,

$$
\left(\int_{B\left(x_{0}, \rho \lambda R\right)} u^{2}(x, 0)\right)^{K} \leq \lambda^{-K} e^{J R^{2}} \int_{B\left(x_{0}, \rho \lambda R / 2\right)} u^{2}(x, 0) .
$$

We now use a chain-of-balls argument similar to that of $\left[9\right.$. Let $x_{k+1}=\left(1-\frac{\rho \lambda^{2}}{8}\right) x_{k}$ for $k=0,1,2, \ldots$. Then by (11),

$$
\left(\int_{B\left(x_{k+1}, \rho \lambda^{2}\left|x_{k+1}\right| / 8\right)} u^{2}(x, 0)\right)^{K} \leq \lambda^{-K} e^{J\left|x_{k}\right|^{2}} \int_{B\left(x_{k}, \rho \lambda^{2}\left|x_{k}\right| / 8\right)} u^{2}(x, 0) \quad k=0,1, \ldots
$$

Let $m=\left[\log \left|x_{0}\right| / \log \frac{8}{8-\rho \lambda^{2}}\right]$. Then $\left|x_{m}\right| \sim 1$; hence

$$
\int_{B\left(x_{m}, \rho \lambda^{2}\left|x_{m}\right| / 8\right)} u^{2}(x, 0) \geq \lambda^{K} e^{-J\left|x_{m}\right|^{2}}\left(\int_{B\left(e_{1}, \rho \lambda^{2} / 8\right)} u^{2}(x, 0)\right)^{K} \geq 1
$$


(we have used (7) in the last inequality). It follows that

$$
\int_{B\left(x_{0}, \rho \lambda^{2}\left|x_{0}\right| / 8\right)} u^{2}(x, 0) \geq \lambda^{\frac{K^{m+1}-K}{K-1}} e^{-\frac{J\left(K^{m}-1\right)}{K-1}\left|x_{0}\right|^{2}},
$$

which, by the choice of $m$, implies

$$
\int_{B\left(x_{0}, \rho \lambda\left|x_{0}\right| / 2\right)} u^{2}(x, 0) \geq e^{-C_{s}\left|x_{0}\right|^{s}}
$$

for some positive constants $s$ and $C_{s}$. The same inequality holds for $u(\cdot, t)$ if $t$ is small so that (7) holds.

2.2.2. Second step. We now use (6) and another Carleman inequality to prove (5). Let $\psi \in C_{c}^{\infty}(0, T)$ be a positive bump function satisfying

$$
\psi(t)=\left\{\begin{array}{cc}
0 & \text { if } t \in\left[0, \frac{T}{8}\right] \cup\left[\frac{7 T}{8}, T\right], \\
4 & t \in\left[\frac{T}{4}, \frac{3 T}{4}\right] .
\end{array}\right.
$$

Let $\delta \in(1,1+\epsilon / 2)$, where $\epsilon$ is the constant in the hypothesis of Theorem 1.1. Let

$$
S_{R, T}:=\left\{(x, t): R^{1 / \delta} \leq|x| \leq R, T / 8<t<7 T / 8\right\} .
$$

Lemma 2.4. Let $G(x, t)=e^{\varphi(x, t)}$, where

$$
\varphi(x, t)=E_{1} R(T-t)|x|+E_{2}\left|x-R \psi(t) e_{1}\right|^{2} .
$$

Here $E_{1} \gtrsim T^{-2}, E_{2} \gtrsim 1$ are constants that may depend on $R$, but $E_{1} / E_{2} \geq 100 / T$ is a fixed constant. Then if $R \geq R_{0}=R_{0}\left(E_{1} / E_{2}, T, \lambda\right)$,

$$
E_{1}^{3} R^{2} \int_{\mathbb{R}_{+}^{n+1}} v^{2} G d x d t+E_{2} \int_{\mathbb{R}_{+}^{n+1}}|\nabla v|^{2} G d x d t \lesssim \int_{\mathbb{R}_{+}^{n+1}}|P v|^{2} G d x d t
$$

for any $v \in C_{c}^{\infty}\left(S_{R, T}\right)$. The implicit constant depends only on $T$ and $\lambda$.

We give a proof of this lemma in the Appendix. Note that in contrast to Lemma 2.2. here the main term in $\varphi$ is $E_{1} R(T-t)|x|$, as $E_{1} \gg E_{2}$. The use of the shift $x-R \psi(t) e_{1}$ originates in a Carleman inequality for Schrödinger equations proved in [4] (see Lemma 3.1 therein).

The next proposition, a corollary of this lemma, is the basis of our bootstrap argument.

Proposition 2.5. Let $u$ be as in Theorem 1.1. Suppose that for some $s \geq 2$, there exist $C_{s}>0$ such that

$$
\int_{T / 4}^{3 T / 4} \int_{R \leq|x| \leq 2 R}\left(u^{2}+|\nabla u|^{2}\right) d x d t \gtrsim \exp \left(-C_{s} R^{s}\right)
$$

for all $R \geq C_{s}$. Let $s_{1}=\max \left\{2, \frac{s-1}{\delta}+1\right\}$, where $1<\delta<1+\frac{\epsilon}{2}$. Then there is $C_{s_{1}}>0$ such that

$$
\int_{0}^{T} \int_{R-1 \leq|x| \leq R}\left(u^{2}+|\nabla u|^{2}\right) d x d t \gtrsim \exp \left(-C_{s_{1}} R^{s_{1}}\right)
$$

for all $R \geq C_{s_{1}}$. 
Proof. Let $v(x, t)=u(x, t) \theta(x, t)$, where $\theta(x, t)=\theta_{1}(x) \theta_{2}\left(x-R \psi(t) e_{1}\right)$, with $\psi$ defined as above, and

$$
\begin{gathered}
\theta_{1}(x)= \begin{cases}0 & \text { if }|x|<R^{1 / \delta} \text { or }|x|>c R, \\
1 & \text { if } R^{1 / \delta}+1 \leq|x| \leq c R-1,\end{cases} \\
\theta_{2}(x)= \begin{cases}0 & \text { if }|x|<2 R, \\
1 & \text { if }|x|>3 R,\end{cases}
\end{gathered}
$$

with $c=2^{-11}$. Clearly, $\operatorname{supp}(v) \subset S_{R, T}$.

We have

$$
\begin{aligned}
|P v| & \leq C(|v|+|\nabla v|)+|u|\left(C|\nabla \theta|+\left|\partial_{t} \theta\right|+|\Delta \theta|\right)+2 \lambda^{-1}|\nabla u||\nabla \theta| \\
& \leq C(|v|+|\nabla v|)+C^{\prime}(|u|+|\nabla u|) \chi_{E},
\end{aligned}
$$

where $E=\operatorname{supp} \nabla \theta$ and $C^{\prime} \leq \lambda^{4} / T$.

Applying the previous Carleman inequality to $v$, we get

$$
\int_{0}^{T} \int_{\mathbb{R}^{n}}\left(v^{2}+|\nabla v|^{2}\right) G d x d t \lesssim \int_{E}\left(|u|^{2}+|\nabla u|^{2}\right) G d x d t .
$$

Since

$$
\begin{aligned}
\inf _{16 R^{1 / \delta}} \leq|x| \leq 32 R^{1 / \delta} & \{G(x, t)\} \geq \exp \left(4 E_{1} T R^{1+\frac{1}{\delta}}+E_{2}\left(4 R-32 R^{1 / \delta}\right)^{2}\right), \\
T / 4 & \leq t \leq 3 T / 4
\end{aligned}
$$

if $E_{1} \geq 2 \cdot 16^{s} C_{s} R^{\frac{s-1}{\delta}-1}$ and $E_{1} / E_{2}=256 / T$, then

$$
\begin{aligned}
& \int_{0}^{T} \int_{\mathbb{R}^{n}}\left(|v|^{2}+|\nabla v|^{2}\right) G d x d t \gtrsim \exp \left(4 E_{1} T R^{1+\frac{1}{\delta}}+E_{2}\left(4 R-32 R^{1 / \delta}\right)^{2}\right. \\
&\left.-16^{s} C_{s} R^{s / \delta}\right) \\
& \geq \exp \left(3 E_{1} T R^{1+\frac{1}{\delta}}+16 E_{2} R^{2}\right)=: \Sigma .
\end{aligned}
$$

The set $E$ is contained in the union of $\left\{R^{1 / \delta} \leq|x| \leq R^{1 / \delta}+1\right\},\left\{2 R \leq\left|x-R \psi(t) e_{1}\right|\right.$ $\leq 3 R\} \cap\{|x| \leq c R\}$ and $\{c R-1 \leq|x| \leq c R\}$. In $\left\{R^{1 / \delta} \leq|x| \leq R^{1 / \delta}+1\right\}$,

$$
G(x, t) \leq \exp \left(2 E_{1} T R^{1+\frac{1}{\delta}}+E_{2}\left(4 R+2 R^{1 / \delta}\right)^{2}\right)
$$

and $|u|+|\nabla u| \lesssim e^{C R^{2 / \delta}}$; hence

$$
\begin{aligned}
& \int_{0}^{T} \int_{R^{1 / \delta} \leq|x| \leq 2 R^{1 / \delta}}\left(|u|^{2}+|\nabla u|^{2}\right) G d x d t \\
& \lesssim \exp \left(2 E_{1} T R^{1+\frac{1}{\delta}}+16 E_{2} R^{2}+20 E_{2} R^{1+\frac{1}{\delta}}+C R^{2 / \delta}\right) \ll \Sigma / 4 .
\end{aligned}
$$

In $\left\{2 R \leq\left|x-R \psi(t) e_{1}\right| \leq 3 R\right\} \cap\{|x| \leq c R\}$,

$$
G(x, t) \leq \exp \left(c^{2} E_{1} T R^{2}+9 E_{2} R^{2}\right) \leq \exp \left(10 E_{2} R^{2}\right) ;
$$

hence

$$
\int_{0}^{T} \int_{2 R \leq\left|x-R \psi(t) e_{1}\right| \leq 3 R,|x| \leq c R}\left(|u|^{2}+|\nabla u|^{2}\right) G d x d t \ll \Sigma / 4
$$


Thus, we conclude that

$$
\Sigma / 4 \leq \int_{0}^{T} \int_{c R-1 \leq|x| \leq c R}\left(|u|^{2}+|\nabla u|^{2}\right) G d x d t .
$$

Since in $\{c R-1 \leq|x| \leq c R\}, G \leq \exp \left(25 E_{2} R^{2}\right)$, we obtain

$$
\int_{0}^{T} \int_{c R-1 \leq|x| \leq c R}\left(|u|^{2}+|\nabla u|^{2}\right) d x d t \geq \exp \left(-9 E_{2} R^{2}\right) .
$$

Recall that we need $E_{1} \geq 2 \cdot 16^{s} C_{s} R^{\frac{s-1}{\delta}-1}$ and $E_{1} \gtrsim T^{-2}$. With the minimum choice $E_{1} \sim \max \left\{1, R^{\frac{s-1}{\delta}-1}\right\}$, we obtain

$$
\int_{0}^{T} \int_{c R-1 \leq|x| \leq c R}\left(|u|^{2}+|\nabla u|^{2}\right) d x d t \geq \exp \left(-C_{s_{1}} R^{s_{1}}\right)
$$

for large $R$. The proposition follows from this.

Proposition 2.6. Suppose $u$ satisfies the assumption of Theorem 1.1. If $u(\cdot, 0) \not \equiv$ 0 , then for any $T \leq 1$, there exist $C_{2}=C_{2}(T, u)>0$ such that

$$
\int_{0}^{T} \int_{R-1 \leq|x| \leq R}\left(u^{2}+|\nabla u|^{2}\right) d x d t \gtrsim \exp \left(-C_{2} R^{2}\right)
$$

for all $R \geq C_{2}$.

Proof. This is a consequence of repeatedly applying the previous proposition. Let $s_{0}=s$, where $s$ is the exponent appearing in (6), and

$$
s_{k+1}=2+\left(\frac{s_{k}-1}{\delta}-1\right)_{+}, \quad k=1,2,3, \ldots .
$$

It is simple to check that there is $k_{0}$ such that $s_{k}=2$ for all $k \geq k_{0}$. Clearly, we can assume that on $[0, T]$, (6) holds. Let $a_{k}=T\left(\frac{1}{2}-2^{k-k_{0}-1}\right)$ and $b_{k}=$ $T\left(\frac{1}{2}+2^{k-k_{0}-1}\right)$. Since

$$
\int_{a_{0}}^{b_{0}} \int_{R<|x|<2 R}|u(x, t)|^{2} d x d t \gtrsim e^{-R^{s}},
$$

the previous proposition (applied to the time interval $\left[a_{1}, b_{1}\right]$ ) shows that

$$
\int_{a_{1}}^{b_{1}} \int_{R-1 \leq|x| \leq R}\left(u^{2}+|\nabla u|^{2}\right) d x d t \gtrsim \exp \left(-C_{s_{1}} R^{s_{1}}\right) \quad \text { if } R \geq C_{s_{1}}
$$

for some positive $C_{s_{1}}$. Induction then shows that for any $k$, there is $C_{s_{k}}>0$ such that

$$
\int_{a_{k}}^{b_{k}} \int_{R-1 \leq|x| \leq R}\left(u^{2}+|\nabla u|^{2}\right) d x d t \gtrsim \exp \left(-C_{s_{k}} R^{s_{k}}\right) \quad \text { if } R \geq C_{s_{1}} .
$$

In particular when $k=k_{0}$ we obtain

$$
\int_{0}^{T} \int_{R-1 \leq|x| \leq R}\left(u^{2}+|\nabla u|^{2}\right) d x d t \gtrsim e^{-C_{2} R^{2}} .
$$




\subsection{Proof of Theorem 1.1.}

Proof. 1. Suppose otherwise $u \not \equiv 0$. We can assume without loss of generality that $u(\cdot, 0) \not \equiv 0$. (If not, we can translate to a time $0<s<1$ such that $u(\cdot, s) \not \equiv 0$. The bounds $|u(x, s)| \lesssim e^{-M|x|^{2}}$ for all $M$ follow from (2).) But then we are in position to apply Proposition 2.6 and obtain a lower bound that contradicts the upper bound of Proposition 2.3 Thus, we must have $u \equiv 0$.

2. Let $T=\rho^{2} /(8 N)$. Inspecting the proof of Proposition 2.3, we see that to obtain the upper bound

$$
|u(x, t)|+|\nabla u(x, t)| \lesssim e^{-M|x|^{2}} \text { in }\left(B_{7 R} \backslash B_{6 R}\right) \times[0, T / 4],
$$

for some $M \geq 2 N$, it suffices to have

$$
\int_{B(x, 1)} u^{2}(y, 0) \leq e^{-2 M|x|^{2}}
$$

for all $x \in B_{2 M R} \backslash B_{R / 2}$. Hence, in order to avoid a contradiction with the lower bound (5), we must have

$$
\sup _{x \in B_{2 M R} \backslash B_{R / 2}} \int_{B(x, 1)} u^{2}(y, 0) \geq e^{-4 M^{2} R^{2}},
$$

if $M \geq 2 \max \left\{N, C_{2}\right\}$ (here $C_{2}$ is the constant appearing in Proposition 2.6). This and (11) together with a chain-of-balls argument show that

$$
\inf _{x \in B_{M R} \backslash B_{R}} \int_{B(x, \rho \lambda R)} u^{2}(y, 0) \geq e^{-M_{1} R^{2}},
$$

for some $M_{1}>0$. Combining this with the doubling inequality (10), we obtain

$$
\inf _{x \in B_{M R} \backslash B_{R}} \int_{B(x, 1)} u^{2}(y, 0) \geq e^{-M_{2} R^{2} \log R} .
$$

These estimates prove the second part of the theorem.

Remark 2.7. As the cutoff functions used in the proof of Theorem 1.1 are radial, the same results and proofs apply to solutions of $|P u| \lesssim|u|+|\nabla u|$ in $\left(\mathbb{R}^{n} \backslash B_{R}\right) \times[0,1]$.

\section{Proof of Theorem 1.2}

The proof of Theorem 1.2 is very similar to that of Theorem 1.1, using anisotropic Carleman inequalities. We use the notation $x=\left(x_{1}, x^{\prime}\right)$. Again, we assume $T=1$.

3.1. Upper bound. For the first step, the same argument as in section 2.1.1 shows that for all $M>0$,

$$
|u(x, t)|+|\nabla u(x, t)| \lesssim e^{C|x|^{2}-M x_{1}^{2}} \text { for all } x \in \mathbb{R}_{+}^{n},
$$

if $0 \leq t \lesssim M^{-1}$. Here $C$ is the constant in the statement of Theorem 1.2, which can be made small by rescaling. Note that now we can only rescale with $R \sim x_{1}$, hence the weaker bound.

For the second step, we will need the next lemma, which is inspired by a Carleman inequality in [6]. To ease notation, we will assume that $a_{\infty}^{1 j}=0$ for $j \neq 1$, where $a_{\infty}^{i j}=\lim _{x \rightarrow \infty} a^{i j}(x, t)$. Otherwise, we will need to replace $\varphi$ in Lemma 3.1 below by

$$
\tilde{\varphi}(x, t)=\varphi\left(x_{1}, B x^{\prime}, t\right)
$$


where $B$ is a positive definite, symmetric $(n-1) \times(n-1)$ matrix, satisfying $\sum_{j \neq 1} B^{i j} a_{\infty}^{1 j}=0$ for $2 \leq i \leq n$. The reader can check that the conclusion of the lemma holds with such a modification of $\varphi$. (We only use $a_{\infty}^{1 j}=0$ to control the term $I_{4}$ in the proof.)

Lemma 3.1. Let $\epsilon$ be the constant in the hypothesis of Theorem 1.2. Let $G(x, t)=$ $e^{\varphi(x, t)}$, where

$$
\varphi(x, t)=-\frac{\lambda\left|x^{\prime}\right|^{2}}{8 s}+\frac{c\left(S^{\alpha}-s^{\alpha}\right)}{s^{\alpha}} x_{1}+b s .
$$

Here $0 \leq c \leq R^{1+\epsilon / 8}$, $\alpha$ and $b \leq \alpha / 4$ are large fixed constants, $s$ is the translated time variable $s=t+1$, and $S=T+1$. Then for large $R$, for any $v \in$ $C_{c}^{\infty}\left(\left\{R \leq x_{1} \leq R^{1+\epsilon / 8}\right\} \times[0, T]\right)$,

$$
\begin{aligned}
& \frac{1}{16} \int_{0}^{T} \int_{\mathbb{R}_{+}^{n}}\left(c R v^{2}+b|\nabla v|^{2}\right) G d x d t \leq \int_{0}^{T} \int_{\mathbb{R}_{+}^{n}}|P v|^{2} G d x d t \\
& \quad+\int_{\mathbb{R}_{+}^{n}}\|\nabla v(x, T)\|^{2} G d x+\int_{\mathbb{R}_{+}^{n}}\left(\left|x^{\prime}\right|^{2}+R^{2+\epsilon}\right) v^{2}(x, 0) G(x, 0) d x \\
& \quad+\int_{\mathbb{R}_{+}^{n}}\left(\left|x^{\prime}\right|^{2}+R^{2+\epsilon}\right) v^{2}(x, T) G(x, T) d x .
\end{aligned}
$$

We give a proof of this lemma in the Appendix.

Proposition 3.2. Suppose that

$$
|u(x, t)|+|\nabla u(x, t)| \lesssim e^{C|x|^{2}-2^{\alpha} x_{1}^{2}} \quad \forall(x, t) \in \mathbb{R}_{+}^{n} \times[0, T] .
$$

Let $d=\frac{2^{\alpha+1}(T+2)}{\alpha T}$, where $\alpha$ is as in the previous lemma. Then for any $M>0$ we have

$$
\int_{0}^{T / 2} \int_{d R<x_{1}<2 d R,\left|x^{\prime}\right|<R}\left(|u|^{2}+|\nabla u|^{2}\right) d x d t \lesssim e^{-M R^{2}} .
$$

Proof. Let

$$
v(x, t)=u(x, t) \theta(x), \text { where } \theta(x)=\theta_{1}(x) \theta_{2}(x),
$$

and

$$
\begin{gathered}
\theta_{1}(x)=\left\{\begin{array}{lc}
0 & \text { if } x_{1}<R-1 \text { or } x_{1}>M R+1, \\
1 & \text { if } R<x_{1}<M R,
\end{array}\right. \\
\theta_{2}(x)=\left\{\begin{array}{rr}
1 & \text { if }\left|x^{\prime}\right|<r, \\
0 & \text { if }\left|x^{\prime}\right|>r+1 .
\end{array}\right.
\end{gathered}
$$

We will now apply the previous lemma with $c=M R$ to the function $v$ and get

$$
\begin{aligned}
& \int_{0}^{T} \int_{\mathbb{R}_{+}^{n}}\left(v^{2}+|\nabla v|^{2}\right) G d x d t \lesssim \int_{E}\left(|u|^{2}+|\nabla u|^{2}\right) G d x d t+\int_{\mathbb{R}_{+}^{n}}\|\nabla v(x, T)\|^{2} G d x \\
& +\int_{\mathbb{R}_{+}^{n}}\left(\left|x^{\prime}\right|^{2}+R^{2+\epsilon}\right) v^{2}(x, 0) G(x, 0) d x+\int_{\mathbb{R}_{+}^{n}}\left(\left|x^{\prime}\right|^{2}+R^{2+\epsilon}\right) v^{2}(x, T) G(x, T) d x,
\end{aligned}
$$

where $E=\operatorname{supp} \nabla \theta \times[0, T]$.

Using (13) and the decay of $u(\cdot, 0)$, we can easily check that the last three integrals on the right hand side are bounded by $R^{2+\epsilon}$, and

$$
\int_{\left\{r<\left|x^{\prime}\right|<r+1, R-1<x_{1}<M R+1\right\} \times[0, T]}\left(|u|^{2}+|\nabla u|^{2}\right) G d x d t \rightarrow 0 \text { as } r \rightarrow \infty .
$$


In $M R<x_{1}<M R+1, G(x, t) \leq e^{-\frac{\lambda\left|x^{\prime}\right|^{2}}{8}+2^{\alpha} x_{1}^{2}+2 b}$. Hence, because of the bound (13),

$$
\int_{0}^{T} \int_{M R<x_{1}<M R+1}\left(|u|^{2}+|\nabla u|^{2}\right) G d x d t \lesssim 1
$$

Furthermore,

$$
\int_{0}^{T} \int_{R-1<x_{1}<R}\left(|u|^{2}+|\nabla u|^{2}\right) G d x d t \lesssim e^{2^{\alpha} M R^{2}} .
$$

Thus, we conclude that

$$
\int_{0}^{T} \int_{\mathbb{R}_{+}^{n}}\left(|v|^{2}+|\nabla v|^{2}\right) G d x d t \lesssim e^{2^{\alpha} M R^{2}}
$$

As in $\left\{x: d R<x_{1}<2 d R,\left|x^{\prime}\right|<R\right\}, u=v$ and $G(x, t) \geq e^{\left(2^{\alpha}+1\right) M R^{2}}$, it follows that

$$
\int_{0}^{T / 2} \int_{d R<x_{1}<2 d R,\left|x^{\prime}\right|<R}\left(|u|^{2}+|\nabla u|^{2}\right) d x d t \lesssim e^{-M R^{2}}
$$

Remark. Using the inequality (11) and a chain-of-balls argument, we can actually take $d$ to be any positive number.

3.2. Lower bound. Assuming $u(\cdot, 0) \not \equiv 0$, the same argument as in section 2.2 gives the lower bound

$$
\int_{B\left(R e_{1}, \rho \lambda R\right)}|u(x, t)|^{2} d x d t \gtrsim e^{-R^{s}} \quad \forall t \in[0, T], \forall R \gtrsim 1
$$

for some $T \leq 1$.

For the second step, we will need another Carleman inequality. Let $\delta \in(1,1+$ $\epsilon / 2)$, where $\epsilon$ is the constant in the hypothesis of Theorem 1.2, and

$$
S_{R, T}:=\left\{(x, t) \in \mathbb{R}_{+}^{n}: R^{1 / \delta} \leq x_{1} \leq R, T / 8<t<7 T / 8\right\} .
$$

Lemma 3.3. Let $G(x, t)=e^{\varphi(x, t)}$, where

$$
\varphi(x, t)=-\frac{\lambda\left|x^{\prime}\right|^{2}}{8 t}+E_{1} R \frac{\left(T^{\alpha}-t^{\alpha}\right)}{t^{\alpha}} x_{1}+E_{2}\left(x_{1}-R \psi(t)\right)^{2}+b E_{2} t,
$$

where $\alpha$ and $b$ are suitable fixed positive constants, $E_{1}, E_{2} \gtrsim 1$ are large constants that may depend on $R$, but $E_{1} / E_{2}$ is a large fixed constant independent of $R$. Then for large $R$,

$$
\int_{\mathbb{R}_{+}^{n+1}} v^{2} G d x d t+\int_{\mathbb{R}_{+}^{n+1}}|\nabla v|^{2} G d x d t \leq \int_{\mathbb{R}_{+}^{n+1}}|P v|^{2} G d x d t
$$

for any $v \in C_{c}^{\infty}\left(S_{R, T}\right)$. Here $\psi$ is as in section 2.2.

We will omit the proof of this lemma as it is almost the same as that of Lemma 3.1. except for the important fact that $E_{1} R \frac{\left(T^{\alpha}-t^{\alpha}\right)}{t^{\alpha}} x_{1}$ is now the dominating term. (This is similar to the relationship between Lemma 2.2 and Lemma 2.4.) 
Proposition 3.4. Let $u$ and $P$ be as in Theorem 1.2. Suppose that for some $s \geq 2$, there are constants $R_{s}, C_{s}>0$ such that

$$
\int_{T / 4}^{3 T / 4} \int_{\substack{R \leq x_{1} \leq 2 R \\\left|x^{\prime}\right| \leq C_{s} R^{s / 2}}}\left(u^{2}+|\nabla u|^{2}\right) d x d t \gtrsim \exp \left(-C_{s} R^{s}\right)
$$

for all $R \geq R_{s}$. Let $s_{1}=\max \left\{2, \frac{s-1}{\delta}+1\right\}$ for some $1<\delta<1+\frac{\epsilon}{2}$. Then there are $R_{s_{1}}, C_{s_{1}}$ such that

$$
\int_{0}^{T} \int \begin{gathered}
R \leq x_{1} \leq 2 R \\
\left|x^{\prime}\right| \leq C_{s_{1}} R^{s_{1} / 2}
\end{gathered} \quad\left(u^{2}+|\nabla u|^{2}\right) d x d t \gtrsim \exp \left(-C_{s_{1}} R^{s_{1}}\right)
$$

for all $R \geq R_{s_{1}}$.

Proof. Let

$$
v(x, t)=u(x, t) \theta(x, t), \text { where } \theta(x, t)=\theta_{1}(x) \theta_{2}\left(x_{1}-R \psi(t)\right) \theta_{3}\left(x^{\prime}\right),
$$

where $\psi$ is defined as before, and

$$
\begin{gathered}
\theta_{1}(x)=\left\{\begin{array}{cc}
0 & \text { if } x_{1}<R^{1 / \delta} \text { or } x_{1}>c R, \\
1 & \text { if } R^{1 / \delta}+1 \leq x_{1} \leq c R-1,
\end{array}\right. \\
\theta_{2}(r)=\left\{\begin{array}{cc}
0 & \text { if } r<2 R, \\
1 & \text { if } r>3 R,
\end{array}\right. \\
\theta_{3}\left(x^{\prime}\right)=\left\{\begin{array}{cc}
0 & \text { if }\left|x^{\prime}\right|>C_{s_{1}} R^{s_{1} / 2}+1, \\
1 & \text { if }\left|x^{\prime}\right|<C_{s_{1}} R^{s_{1} / 2},
\end{array}\right.
\end{gathered}
$$

where $c$ and $C_{s_{1}}$ are positive constants to be chosen. It is clear that $\operatorname{supp}(v) \subset S_{R, T}$.

Applying the previous Carleman inequality to $v$, we get

$$
\int_{\mathbb{R}_{+}^{n+1}}\left(v^{2}+|\nabla v|^{2}\right) G d x d t \lesssim \int_{E}\left(|u|^{2}+|\nabla u|^{2}\right) G d x d t
$$

where $E=\operatorname{supp} \nabla \theta$.

Because in the set $\left\{x: 10^{\alpha} R^{1 / \delta} \leq x_{1} \leq 2 \cdot 10^{\alpha} R^{1 / \delta},\left|x^{\prime}\right| \leq C_{s}\left(10^{\alpha} R^{1 / \delta}\right)^{s / 2}\right\} \times$ $\left[\frac{T}{4}, \frac{3 T}{4}\right]$,

$$
G(x, t) \geq \exp \left(-D_{s}^{\prime} R^{s / \delta}+10^{\alpha} E_{1} R^{1+\frac{1}{\delta}}+E_{2}\left(4 R-2 \cdot 10^{\alpha} R^{1 / \delta}\right)^{2}\right),
$$

we have

$$
\begin{aligned}
& \int_{\mathbb{R}_{+}^{n+1}}\left(|v|^{2}+|\nabla v|^{2}\right) G d x d t \gtrsim \exp ( 10^{\alpha} E_{1} R^{1+\frac{1}{\delta}}+E_{2}\left(4 R-2 \cdot 10^{\alpha} R^{1 / \delta}\right)^{2} \\
&\left.-C_{s} R^{s / \delta}-D_{s}^{\prime} R^{s / \delta}\right) \\
& \gtrsim \exp \left(9^{\alpha} E_{1} R^{1+\frac{1}{\delta}}+16 E_{2} R^{2}\right)=: \Sigma
\end{aligned}
$$

if $E_{1} / R^{\frac{s-1}{\delta}-1}$ and $E_{1} / E_{2}$ are large enough. 
In $\left\{R^{1 / \delta} \leq x_{1} \leq R^{1 / \delta}+1\right\}$,

$$
G(x, t) \leq \exp \left(-\frac{\lambda\left|x^{\prime}\right|^{2}}{8}+8^{\alpha} E_{1} R^{1+\frac{1}{\delta}}+16 E_{2} R^{2}\right),
$$

so using the bound (13) we get

$$
\int_{0}^{T} \int_{R^{1 / \delta} \leq x_{1} \leq 2 R^{1 / \delta}}\left(|u|^{2}+|\nabla u|^{2}\right) G d x d t \lesssim \exp \left(8^{\alpha} E_{1} R^{1+\frac{1}{\delta}}+16 E_{2} R^{2}\right) \ll \Sigma .
$$

In $\left\{2 R \leq\left|x_{1}-R \psi(t)\right| \leq 3 R\right\} \cap\left\{x_{1} \leq c R\right\}$,

$$
G(x, t) \leq \exp \left(-\frac{\lambda\left|x^{\prime}\right|^{2}}{8}+c 8^{\alpha} E_{1} R^{2}+9 E_{2} R^{2}\right) .
$$

Hence, if $c$ is chosen to be small enough,

$$
\int_{0}^{T} \int_{2 R \leq\left|x_{1}-R \psi(t)\right| \leq 3 R, x_{1} \leq c R}\left(|u|^{2}+|\nabla u|^{2}\right) G d x d t \lesssim \exp \left(10 E_{2} R^{2}\right) \ll \Sigma .
$$

In $\left\{C_{s_{1}} R^{s_{1} / 2} \leq\left|x^{\prime}\right| \leq C_{s_{1}} R^{s_{1} / 2}+1\right\}$,

$$
G(x, t) \leq \exp \left(-\lambda C_{s_{1}}^{2} R^{s_{1}} / 8+c 8^{\alpha} E_{1} R^{2}+16 E_{2} R^{2}\right) .
$$

Note that by our choice of $E_{1}$ and $E_{2}, E_{1} R^{2} \sim E_{2} R^{2} \sim R^{s_{1}}$, so if we choose $C_{s_{1}}$ large enough,

$$
\int_{0}^{T} \int_{x_{1}<c R, C_{s_{1}} R^{s_{1} / 2} \leq\left|x^{\prime}\right| \leq C_{s_{1}} R^{s_{1} / 2}+1}\left(|u|^{2}+|\nabla u|^{2}\right) G d x d t \lesssim 1 .
$$

Thus, we conclude that

$$
\Sigma \lesssim \int_{0}^{T} \int_{c R<x_{1}<c R+1,\left|x^{\prime}\right| \leq C_{s_{1}} R^{s_{1} / 2}}\left(|u|^{2}+|\nabla u|^{2}\right) G d x d t .
$$

Since in $\left\{c R<x_{1}<c R+1,\left|x^{\prime}\right| \leq C_{s_{1}} R^{s_{1} / 2}\right\}, G \leq \exp \left(K R^{s_{1}}\right)$, we obtain

$$
\begin{gathered}
\int_{0}^{T} \int_{c R \leq x_{1} \leq c R+1}\left(|u|^{2}+|\nabla u|^{2}\right) d x d t \geq \exp \left(-K R^{s_{1}}\right) . \\
\left|x^{\prime}\right| \leq C_{s_{1}} R^{s_{1} / 2}
\end{gathered}
$$

The proposition follows immediately from this.

Proposition 3.5. Let $u$ and $P$ be as in Theorem 1.2. If $u(\cdot, 0) \not \equiv 0$, then for any $T \leq 1$, there exist $C_{2}=C_{2}(T, u)>0$ such that

$$
\int_{0}^{T} \int_{\substack{R \leq x_{1} \leq 2 R \\\left|x^{\prime}\right| \leq C_{2} R}}\left(u^{2}+|\nabla u|^{2}\right) d x d t \gtrsim \exp \left(-C_{2} R^{2}\right)
$$

for all $R \geq C_{2}$.

Proof. The proof is similar to that of Proposition 2.6, using Proposition 3.4 instead of Proposition 2.5. We omit the details.

Using Proposition 3.2 (see also the remark after it) and Proposition 3.5, the proof of Theorem 1.2 is identical to that of Theorem 1.1. We omit the details. 


\section{Appendix}

4.1. Some auxiliary lemmas. The first lemma is a standard estimate for solutions of parabolic inequalities; we refer to [10].

Lemma 4.1. Suppose that in $\Omega^{*}:=B(0,2) \times\left[0,2 R^{-2}\right]$, the following inequality holds:

$$
|P v| \leq R^{2}|v|+R|\nabla v|
$$

Then

$$
\|v\|_{L^{\infty}(\Omega)}+\|\nabla v\|_{L^{\infty}(\Omega)} \leq C_{n} R^{c}\|v\|_{L^{2}\left(\Omega^{*}\right)},
$$

where $\Omega=B(0,1) \times\left[0, R^{-2}\right]$ and $c$ is a constant depending only on $n$.

The next two lemmas are from [2] (see also [3]).

Lemma 4.2. For $\rho \in(0,1 / 2)$, there is a constant $N_{\rho}>0$ such that if

$$
|P v| \leq R^{2}|v|+R|\nabla v|
$$

in $\Omega^{*}:=B(0,2) \times\left[0,2 R^{-2}\right]$, then

$$
\int_{B(0, \rho)} v^{2}(x, 0) d x \leq N_{\rho} \int_{B(0,2 \rho)} v^{2}(x, t) d x
$$

for all

$$
0 \leq t \leq N_{\rho}^{-1} \min \left\{R^{-2}, 1 / \log \left(\frac{N_{\rho} \int_{\Omega^{*}} v^{2}(x, 0) d x d t}{\int_{B(0, \rho)} v^{2}(x, 0) d x}\right)\right\} .
$$

Lemma 4.3. Suppose $v \in C_{c}^{\infty}\left(\mathbb{R}^{n}\right)$ such that for some $C>1$,

$$
2 a \int_{\mathbb{R}^{n}}|\nabla v|^{2} e^{-|x|^{2} / 4 a} d x+\frac{n}{2} \int_{\mathbb{R}^{n}} v^{2} e^{-|x|^{2} / 4 a} d x \leq C \int_{\mathbb{R}^{n}} v^{2} e^{-|x|^{2} / 4 a} d x,
$$

for all $0<a \leq 1 /(12 C)$. Then

$$
\int_{B(0,2 r)} v^{2} d x \leq e^{32 C} \int_{B(0, r)} v^{2} d x
$$

for all $0 \leq r \leq 1 / 2$.

4.2. Proof of the Carleman inequalities. In this section we will prove the Carleman inequalities that were used in the proofs of Theorems 1.1 and 1.2. We will use the following notation:

$$
\begin{gathered}
\Delta v=\operatorname{div}(A \nabla v), \\
\|\nabla v(x, t)\|=\langle A(x, t) \nabla v(x, t), \nabla v(x, t)\rangle^{1 / 2} .
\end{gathered}
$$

We recall the following lemma of [7] (see also [8, [2]).

Lemma 4.4. Suppose $\sigma(t): \mathbb{R}_{+} \rightarrow \mathbb{R}_{+}$is a smooth function, $\alpha$ is a real number, $F$ and $G$ are differentiable functions, and $G$ is positive. Then the following identity 
holds for $v \in C_{c}^{2}\left(\mathbb{R}^{n} \times[0, T]\right)$ :

$$
\begin{aligned}
& 2 \int_{\mathbb{R}_{+}^{n+1}} \frac{\sigma^{1-\alpha}}{\sigma^{\prime}} w^{2} G d x d t+\frac{1}{2} \int_{\mathbb{R}_{+}^{n+1}} \frac{\sigma^{1-\alpha}}{\sigma^{\prime}} v^{2} M G d x d t \\
& \quad-\frac{\alpha}{2} \int_{\mathbb{R}_{+}^{n+1}} \sigma^{-\alpha} v^{2}\left(\frac{\partial_{t} G-\Delta G}{G}-F\right) G d x d t \\
& \quad+\int_{\mathbb{R}_{+}^{n+1}} \frac{\sigma^{1-\alpha}}{\sigma^{\prime}}\left[\left(\log \frac{\sigma}{\sigma^{\prime}}\right)^{\prime}+\frac{\partial_{t} G-\Delta G}{G}-F\right]\|\nabla v\|^{2} G d x d t \\
& \quad+2 \int_{\mathbb{R}_{+}^{n+1}} \frac{\sigma^{1-\alpha}}{\sigma^{\prime}}\left\langle D_{G} \nabla v, \nabla v\right\rangle G d x d t-\int_{\mathbb{R}_{+}^{n+1}} \frac{\sigma^{1-\alpha}}{\sigma^{\prime}} v\langle A \nabla v, \nabla F\rangle G d x d t \\
& \quad=2 \int_{\mathbb{R}_{+}^{n+1}} \frac{\sigma^{1-\alpha}}{\sigma^{\prime}} w P v G d x d t+\int_{\mathbb{R}^{n} \times\{T\}} \frac{\sigma^{1-\alpha}}{\sigma^{\prime}}\|\nabla v\|^{2} G d x \\
& \quad-\int_{\mathbb{R}^{n} \times\{0\}} \frac{\sigma^{1-\alpha}}{\sigma^{\prime}}\|\nabla v\|^{2} G d x+\frac{1}{2} \int_{\mathbb{R}^{n} \times\{T\}} \frac{\sigma^{1-\alpha}}{\sigma^{\prime}} v^{2}\left(F-\frac{\alpha \sigma^{\prime}}{\sigma}\right) G d x \\
& \quad-\frac{1}{2} \int_{\mathbb{R}^{n} \times\{0\}} \frac{\sigma^{1-\alpha}}{\sigma^{\prime}} v^{2}\left(F-\frac{\alpha \sigma^{\prime}}{\sigma}\right) G d x,
\end{aligned}
$$

where

$$
\begin{gathered}
w=\partial_{t} v-\langle A \nabla \log G, \nabla v\rangle+\frac{F v}{2}-\frac{\alpha \sigma^{\prime}}{2 \sigma} v, \\
M=\left(\log \frac{\sigma}{\sigma^{\prime}}\right)^{\prime} F+\partial_{t} F+F\left(\frac{\partial_{t} G-\Delta G}{G}-F\right)-\langle A \nabla \log G, \nabla F\rangle,
\end{gathered}
$$

and

$$
D_{G}^{i j}=a^{i l} \partial_{k l}(\log G) a^{k j}+\frac{\partial_{l}(\log G)}{2}\left[a^{k j} \partial_{k} a^{i l}+a^{k i} \partial_{k} a^{j l}-a^{k l} \partial_{k} a^{i j}\right]+\frac{1}{2} \partial_{t} a^{i j} .
$$

We will first derive a corollary of this lemma which will be used to prove all of our Carleman inequalities. Letting $\alpha=0$ and $\sigma(t)=e^{t}$ in Lemma 4.4 we obtain the following identity for $v \in C_{c}^{2}\left(\mathbb{R}^{n} \times[0, T]\right)$ :

$$
\begin{aligned}
& 2 \int_{\mathbb{R}_{+}^{n+1}} w^{2} G d x d t+\frac{1}{2} \int_{\mathbb{R}_{+}^{n+1}} v^{2} M G d x d t-\int_{\mathbb{R}_{+}^{n+1}} v\langle A \nabla v, \nabla F\rangle G d x d t \\
& +\int_{\mathbb{R}_{+}^{n+1}}\|\nabla v\|^{2}\left(\frac{\partial_{t} G-\Delta G}{G}-F\right) G d x d t+2 \int_{\mathbb{R}_{+}^{n+1}}\left\langle D_{G} \nabla v, \nabla v\right\rangle G d x d t \\
(14)= & 2 \int_{\mathbb{R}_{+}^{n+1}} w P v G d x d t+\int_{\mathbb{R}^{n}}\|\nabla v(x, T)\|^{2} G d x-\int_{\mathbb{R}^{n}}\|\nabla v(x, 0)\|^{2} G d x \\
& +\frac{1}{2} \int_{\mathbb{R}^{n}} v^{2}(x, T) F G d x-\frac{1}{2} \int_{\mathbb{R}^{n}} v^{2}(x, 0) F G d x,
\end{aligned}
$$

where

$$
M=\partial_{t} F+F\left(\frac{\partial_{t} G-\Delta G}{G}-F\right)-\langle A \nabla F, \nabla \log G\rangle
$$


Note that if $\nabla F$ is differentiable, we can integrate by parts to obtain

$$
\begin{aligned}
-\int_{\mathbb{R}_{+}^{n+1}} v\langle A \nabla v, \nabla F\rangle G d x d t & =\frac{1}{2} \int_{\mathbb{R}_{+}^{n+1}} v^{2} \Delta F G d x d t \\
& +\frac{1}{2} \int_{\mathbb{R}_{+}^{n+1}} v^{2}\langle A \nabla F, \nabla \log G\rangle G d x d t .
\end{aligned}
$$

Then this term can be combined with the second term of the left hand side. However, in our applications, $\nabla F$ might not be differentiable, so we approximate $F$ by some $C^{2}$ function $F_{0}$ and use the above identity with $F_{0}$ in place of $F$. Then, using Cauchy-Schwarz, we arrive at the following lemma.

Lemma 4.5. Suppose $v \in C_{c}^{2}\left(\mathbb{R}^{n} \times[0, T]\right)$. Then

$$
\begin{aligned}
& \frac{1}{2} \int_{\mathbb{R}_{+}^{n+1}} v^{2} M_{0} G d x d t+\int_{\mathbb{R}_{+}^{n+1}}\left[2\left\langle D_{G} \nabla v, \nabla v\right\rangle+\|\nabla v\|^{2}\left(\frac{\partial_{t} G-\Delta G}{G}-F\right)\right] G d x d t \\
& -\int_{\mathbb{R}_{+}^{n+1}} v\left\langle A \nabla v, \nabla\left(F-F_{0}\right)\right\rangle G d x d t \leq \int_{\mathbb{R}_{+}^{n+1}}|P v|^{2} G d x d t+\int_{\mathbb{R}^{n}}\|\nabla v(x, T)\|^{2} G d x \\
& -\int_{\mathbb{R}^{n}}\|\nabla v(x, 0)\|^{2} G d x+\frac{1}{2} \int_{\mathbb{R}^{n}} v^{2}(x, T) F G d x-\frac{1}{2} \int_{\mathbb{R}^{n}} v^{2}(x, 0) F G d x,
\end{aligned}
$$

where

$$
M_{0}=\partial_{t} F+F\left(\frac{\partial_{t} G-\Delta G}{G}-F\right)+\Delta F_{0}-\left\langle A \nabla\left(F-F_{0}\right), \nabla \log G\right\rangle
$$

and

$$
D_{G}^{i j}=a^{i l} \partial_{k l}(\log G) a^{k j}+\frac{\partial_{l}(\log G)}{2}\left[a^{k j} \partial_{k} a^{i l}+a^{k i} \partial_{k} a^{j l}-a^{k l} \partial_{k} a^{i j}\right]+\frac{1}{2} \partial_{t} a^{i j} .
$$

We will now prove our Carleman inequalities using Lemma 4.5 .

Proof of Lemma 2.2. As $\operatorname{supp} v \subset\left\{R \leq|x| \leq R^{1+\epsilon / 8}\right\} \times[0, T]$, we will assume that $R \leq|x| \leq R^{1+\epsilon / 8}$ in all the computations below.

Since $\nabla^{2} \varphi \geq \mathrm{Id},|\nabla \log G| \leq R^{1+\epsilon / 8}$, and $\left|\nabla a^{i j}(x, t)\right| \leq R^{-1-\epsilon}$, it follows that $D_{G} \geq \frac{\lambda^{2}}{2}$ Id for large $R$. To make the gradient term (i.e. the second term in (15) positive, we will choose $F$ satisfying

$$
\left|\frac{\partial_{t} G-\Delta G}{G}-F\right| \leq \lambda^{4} / 2
$$

so that

$$
2\left\langle D_{G} \nabla v, \nabla v\right\rangle+\|\nabla v\|^{2}\left(\frac{\partial_{t} G-\Delta G}{G}-F\right) \geq \frac{\lambda^{2}}{2}|\nabla v|^{2} .
$$

Let $\varphi(x, t)=c(T-t)|x|+|x|^{2}$. Then

$$
\begin{aligned}
\frac{\partial_{t} G-\Delta G}{G}= & \partial_{t} \varphi-\Delta \varphi-a^{i j} \partial_{i} \varphi \partial_{j} \varphi \\
= & -c|x|-x_{j} \partial_{i} a^{i j}(x, t)\left(c(T-t)|x|^{-1}+2\right) \\
& -a^{i j}(x, t) x_{i} x_{j}\left(c(T-t)|x|^{-1}+2\right)^{2} \\
& -a^{i j}(x, t)\left[\delta_{i j}\left(c(T-t)|x|^{-1}+2\right)-c(T-t) x_{i} x_{j}|x|^{-3}\right] .
\end{aligned}
$$


As the second term is of order $O\left(R^{-7 \epsilon / 8}\right)$, if we let

$$
\begin{aligned}
F(x, t) & =-c|x|+\frac{\lambda^{4}}{3}-a^{i j}(x, t) x_{i} x_{j}\left(c(T-t)|x|^{-1}+2\right)^{2} \\
& -a^{i j}(x, t)\left[\delta_{i j}\left(c(T-t)|x|^{-1}+2\right)-c(T-t) x_{i} x_{j}|x|^{-3}\right],
\end{aligned}
$$

then (16) is satisfied. Moreover,

$$
-R^{2+\epsilon / 4} \lesssim F \lesssim-R^{2}, \quad-\frac{\lambda^{4}}{2} \leq \frac{\partial_{t} G-\Delta G}{G}-F \leq-\frac{\lambda^{4}}{4} .
$$

We have

$$
\begin{aligned}
\partial_{t} F(x, t)= & -\partial_{t} a^{i j}(x, t) x_{i} x_{j}\left(c(T-t)|x|^{-1}+2\right)^{2} \\
& +2 c a^{i j}(x, t) x_{i} x_{j}|x|^{-1}\left(c(T-t)|x|^{-1}+2\right) \\
& -\partial_{t}\left\{a^{i j}(x, t)\left[\delta_{i j}\left(c(T-t)|x|^{-1}+2\right)-c(T-t) x_{i} x_{j}|x|^{-3}\right]\right\} .
\end{aligned}
$$

The second term on the right hand side is positive by ellipticity of $\left\{a^{i j}\right\}$. Noting that the last terms of $F$ and $\partial_{t} F$ are $O\left(R^{\epsilon / 8}\right)$, we get

$$
\begin{aligned}
\partial_{t} F & +F\left(\frac{\partial_{t} G-\Delta G}{G}-F\right) \\
& \geq-\left(\frac{\partial_{t} G-\Delta G}{G}-F\right) a^{i j}(x, t) x_{i} x_{j}\left(c(T-t)|x|^{-1}+2\right)^{2} \\
& \quad-\partial_{t} a^{i j}(x, t) a^{i j}(x, t) x_{i} x_{j}\left(c(T-t)|x|^{-1}+2\right)^{2}+O\left(R^{\epsilon / 8}\right) \\
& \gtrsim R^{2}
\end{aligned}
$$

(note that $\left|\partial_{t} a^{i j}(x, t)\right| \leq C \leq \lambda^{5} / 100$ ).

For the approximation $F_{0}$ of $F$, we choose

$$
\begin{aligned}
F_{0}(x, t)= & -c|x|+\frac{\lambda^{4}}{3}-a^{i j}(X, t) x_{i} x_{j}\left(c(T-t)|x|^{-1}+2\right)^{2} \\
& -a^{i j}(X, t)\left[\delta_{i j}\left(c(T-t)|x|^{-1}+2\right)-c(T-t) x_{i} x_{j}|x|^{-3}\right],
\end{aligned}
$$

where $X=(2 R, 0, \ldots, 0)$.

As

$$
\left|a^{i j}(x, t)-a^{i j}(X, t)\right|=O\left(R^{-7 \epsilon / 8}\right), \quad\left|\nabla a^{i j}(x, t)\right|=O\left(R^{-1-\epsilon}\right)
$$

and

$$
\begin{aligned}
& x_{i} x_{j}\left(c(T-t)|x|^{-1}+2\right)^{2}=O\left(R^{2+\epsilon / 4}\right), \\
& \nabla\left(x_{i} x_{j}\left(c(T-t)|x|^{-1}+2\right)^{2}\right)=O\left(R^{1+\epsilon / 4}\right) \\
& \delta_{i j}\left(c(T-t)|x|^{-1}+2\right)-c(T-t) x_{i} x_{j}|x|^{-3}=O\left(R^{\epsilon / 8}\right), \\
& \nabla\left(\delta_{i j}\left(c(T-t)|x|^{-1}+2\right)-c(T-t) x_{i} x_{j}|x|^{-3}\right)=O\left(R^{-1+\epsilon / 8}\right),
\end{aligned}
$$

we have

$$
\nabla\left(F-F_{0}\right)=O\left(R^{1-5 \epsilon / 8}\right) .
$$


An easy computation shows that

$$
\Delta F_{0}=O\left(R^{\epsilon / 4}\right) .
$$

Thus,

$$
M_{0}=\partial_{t} F+F\left(\frac{\partial_{t} G-\Delta G}{G}-F\right)+\Delta F_{0}-\left\langle A \nabla\left(F-F_{0}\right), \nabla \log G\right\rangle \gtrsim R^{2} .
$$

Finally, we can use Cauchy-Schwarz to control the remaining term as follows:

$$
\left|\int_{0}^{T} \int_{\mathbb{R}^{n}} v\left\langle A \nabla v, \nabla\left(F-F_{0}\right)\right\rangle G d x d t\right| \leq \frac{M_{0}}{4} \int_{\mathbb{R}^{n}} v^{2} G d x d t+\frac{\lambda^{2}}{4} \int_{\mathbb{R}^{n}}|\nabla v|^{2} G d x d t .
$$

This shows that the left hand side of (4.5) is greater than

$$
\frac{R^{2}}{4} \int_{\mathbb{R}^{n}} v^{2} G d x d t+\frac{\lambda^{2}}{4} \int_{\mathbb{R}^{n}}|\nabla v|^{2} G d x d t .
$$

In our case, $F<0$, so the third and fourth terms on the right hand side of (4.5) are negative. Thus, the lemma is proved.

Proof of Lemma 2.4. As $\nabla^{2} \varphi \geq 2 E_{2} \mathrm{Id}$, the first term in $D_{G}$ is at least $2 \lambda^{2} E_{2} \mathrm{Id}$. The middle three terms of $D_{G}$ are $O\left(E_{1} R^{1-\frac{1+\epsilon}{\delta}}\right)$, and the last term is bounded by $C \leq \lambda^{4}$. Thus, $D_{G} \geq \lambda^{2} E_{2}$ Id. To make the gradient term positive, we will chose $F$ satisfying

$$
\left|\frac{\partial_{t} G-\Delta G}{G}-F\right| \leq \lambda^{4} E_{2}
$$

so that then

$$
2\left\langle D_{G} \nabla v, \nabla v\right\rangle+\|\nabla v\|^{2}\left(\frac{\partial_{t} G-\Delta G}{G}-F\right) \geq \lambda^{2} E_{2}|\nabla v|^{2} .
$$

Let $\widetilde{x}=x-R \psi(t) e_{1}$. Then we have

$$
\begin{aligned}
\frac{\partial_{t} G-\Delta G}{G}= & \partial_{t} \varphi-\Delta \varphi-a^{i j} \partial_{i} \varphi \partial_{j} \varphi \\
= & -E_{1} R|x|-2 E_{2} R \psi^{\prime}(t)\left(x_{1}-R \psi(t)\right) \\
& -\partial_{i} a^{i j}(x, t)\left(E_{1} R(T-t) \frac{x_{j}}{|x|}+2 E_{2} \widetilde{x_{j}}\right) \\
& -a^{i j}(x, t)\left(E_{1} R(T-t) \frac{x_{i}}{|x|}+2 E_{2} \widetilde{x_{i}}\right)\left(E_{1} R(T-t) \frac{x_{j}}{|x|}+2 E_{2} \widetilde{x_{j}}\right) \\
& -a^{i j}(x, t)\left[-E_{1} R(T-t) \frac{x_{i} x_{j}}{|x|^{3}}+\delta_{i j}\left(E_{1} R(T-t)|x|^{-1}+2 E_{2}\right)\right] .
\end{aligned}
$$

Note that in $S_{R, T}$ we have

$$
\left|\nabla a^{i j}(x, t)\right| \leq\langle x\rangle^{-1-\epsilon} \leq R^{-(1+\epsilon) / \delta},
$$

hence

$$
\left|\partial_{i} a^{i j}(x, t)\left(E_{1} R(T-t) \frac{x_{j}}{|x|}+2 E_{2} \widetilde{x_{j}}\right)\right| \lesssim E_{1} R^{1-\frac{1+\epsilon}{\delta}} .
$$


Thus, we choose

$$
\begin{aligned}
F(x, t)= & -E_{1} R|x|-2 E_{2} R \psi^{\prime}(t)\left(x_{1}-R \psi(t)\right)+\lambda^{4} E_{2} / 2 \\
& -a^{i j}(x, t)\left(E_{1} R(T-t) \frac{x_{j}}{|x|}+2 E_{2} \widetilde{x_{j}}\right)\left(E_{1} R(T-t) \frac{x_{i}}{|x|}+2 E_{2} \widetilde{x_{i}}\right) \\
& -a^{i j}(x, t)\left[-E_{1} R(T-t) \frac{x_{i} x_{j}}{|x|^{3}}+\delta_{i j}\left(E_{1} R(T-t)|x|^{-1}+2 E_{2}\right)\right] .
\end{aligned}
$$

Also, let

$$
\begin{aligned}
F_{0}(x, t)= & -E_{1} R|x|-2 E_{2} R \psi^{\prime}(t)\left(x_{1}-R \psi(t)\right)+\lambda^{4} E_{2} / 2 \\
& -a^{i j}(X, t)\left(E_{1} R(T-t) \frac{x_{j}}{|x|}+2 E_{2} \widetilde{x_{j}}\right)\left(E_{1} R(T-t) \frac{x_{i}}{|x|}+2 E_{2} \widetilde{x_{i}}\right) \\
& -a^{i j}(X, t)\left[-E_{1} R(T-t) \frac{x_{i} x_{j}}{|x|^{3}}+\delta_{i j}\left(E_{1} R(T-t)|x|^{-1}+2 E_{2}\right)\right],
\end{aligned}
$$

where $X=\left(2 R^{1 / \delta}, 0, \ldots, 0\right)$.

In the support of $v, T-t \geq T / 8$, and $|\tilde{x}| \leq 5 R$, so by ellipticity of $\left\{a^{i j}\right\}$,

$$
\begin{aligned}
& -a^{i j}\left(E_{1} R(T-t) \frac{x_{j}}{|x|}+2 E_{2} \widetilde{x_{j}}\right)\left(E_{1} R(T-t) \frac{x_{i}}{|x|}+2 E_{2} \widetilde{x_{i}}\right) \\
& \leq-\lambda\left|E_{1} R(T-t) \frac{x}{|x|}-2 E_{2} \tilde{x}\right|^{2} \lesssim-T^{2} E_{1}^{2} R^{2} .
\end{aligned}
$$

The other terms in $F$ are bounded by $E_{1} R^{2}, E_{2} R^{2} / T, E_{2}$, and $E_{1} T R^{1-\frac{1}{\delta}}$. Hence, for large $R$,

$$
F \lesssim-E_{1}^{2} T^{2} R^{2} \text { and } F\left(\frac{\partial_{t} G-\Delta G}{G}-F\right) \gtrsim E_{1}^{2} E_{2} R^{2} .
$$

It is easy to check that

$$
\begin{aligned}
\partial_{t} F & =O\left(E_{1}^{2} R^{2}\right), \\
\Delta F_{0} & =O\left(E_{1}^{2} R^{2-\frac{2}{\delta}}\right),
\end{aligned}
$$

which is smaller than $F\left(\frac{\partial_{t} G-\Delta G}{G}-F\right)$ provided $E_{2} \gg 1$. Using $\left|a^{i j}(x, t)-a^{i j}(X, t)\right|$ $\lesssim R^{1-\frac{1+\epsilon}{\delta}}$ and $\left|\nabla\left(a^{i j}(x, t)-a^{i j}(X, t)\right)\right|=R^{-\frac{1+\epsilon}{\delta}}$ in $S_{R, T}$, we get

$$
\left|\nabla\left(F-F_{0}\right)\right| \lesssim R^{3-\frac{2+\epsilon}{\delta}} E_{1}^{2}
$$

hence

$$
\left|\left\langle A \nabla\left(F-F_{0}\right), \nabla \log G\right\rangle\right| \lesssim R^{4-\frac{2+\epsilon}{\delta}} E_{1}^{3} \ll E_{1}^{2} E_{2} R^{2}
$$

for large $R$, as $\delta<1+\frac{\epsilon}{2}$.

Putting together these estimates, we obtain

$$
M_{0}=\partial_{t} F+\Delta F_{0}+F\left(\frac{\partial_{t} G-\Delta G}{G}-F\right)-\left\langle A \nabla\left(F-F_{0}\right), \nabla \log G\right\rangle \geq E_{1}^{2} E_{2} R^{2} .
$$

Finally, since

$$
M_{0} E_{2} \gg R^{6-\frac{2(2+\epsilon)}{\delta}} E_{1}^{4} \gtrsim\left|\nabla\left(F-F_{0}\right)\right|^{2}
$$


we can control the remaining term by Cauchy-Schwarz,

$$
\left|\int_{\mathbb{R}_{+}^{n+1}} u\left\langle A \nabla u, \nabla\left(F-F_{0}\right)\right\rangle G d x d t\right| \leq \frac{M_{0}}{2} \int u^{2} G d x d t+\frac{\lambda^{2} E_{2}}{8} \int|\nabla u|^{2} G d x d t .
$$

Thus, the lemma is proved.

Proof of Lemma 3.1. As $\nabla^{2} \varphi \geq-\frac{\lambda}{8} \operatorname{Id}$ and

$$
|\nabla \log G|\left|\nabla a^{i j}\right|=O\left(|x|^{-7 \epsilon / 8}\right), \quad\left|\partial_{t} a^{i j}\right| \leq C \leq \lambda^{4} / 100,
$$

it follows that if

$$
H:=\frac{\partial_{t} G-\Delta G}{G}-F \geq \frac{1}{\lambda}
$$

then the gradient term is positive. We have

$$
\begin{aligned}
\frac{\partial_{t} G-\Delta G}{G}= & \frac{\lambda}{16 s^{2}} \sum_{i, j \neq 1}\left(2 \delta_{i j}-\lambda a^{i j}(x, t)\right) x_{i} x_{j}-\frac{c \alpha S^{\alpha} x_{1}}{s^{\alpha+1}}+b \\
& -a^{11}(x, t) \frac{c^{2}\left(S^{\alpha}-s^{\alpha}\right)^{2}}{s^{2 \alpha}}-\sum_{j \neq 1} a^{1 j}(x, t) \frac{\lambda x_{j}}{2 s} \frac{c\left(S^{\alpha}-s^{\alpha}\right)}{s^{\alpha}} \\
& -\partial_{i}\left(a^{i j} \partial_{j} \varphi\right) .
\end{aligned}
$$

Since $c \leq x_{1}^{1+\epsilon / 8}$, from the decay of $\nabla a^{i j}$ it follows that $\left|\partial_{i}\left(a^{i j} \partial_{j} \varphi\right)\right| \lesssim 1$. If we choose

$$
\begin{aligned}
F(x, t)= & \frac{\lambda}{16 s^{2}} \sum_{i, j \neq 1}\left(2 \delta_{i j}-\lambda a^{i j}(x, t)\right) x_{i} x_{j}-\frac{c \alpha S^{\alpha} x_{1}}{s^{\alpha+1}} \\
& -a^{11}(x, t) \frac{c^{2}\left(S^{\alpha}-s^{\alpha}\right)^{2}}{s^{2 \alpha}}-\sum_{j \neq 1} a^{1 j}(x, t) \frac{\lambda x_{j}}{2 s} \frac{c\left(S^{\alpha}-s^{\alpha}\right)}{s^{\alpha}}
\end{aligned}
$$

then for large $b$,

$$
2 b \geq H \geq b / 2,
$$

implying the positivity of the gradient term.

Consider four terms of $\left(H+\partial_{t}\right) F$ corresponding to four terms of $F$.

(1) $I_{1}:=\left(\frac{1}{2} H s-1\right) \frac{\lambda}{8 s^{3}} \sum_{i, j \neq 1}\left(2 \delta_{i j}-\lambda a^{i j}(X, t)\right) x_{i} x_{j}-\sum_{i, j \neq 1} \partial_{t} a^{i j} x_{i} x_{j} \geq$ $b\left|x^{\prime}\right|^{2}$ for large $b$.

$$
I_{2}:=\left(\frac{\alpha+1}{s}-H\right) \frac{c \alpha S^{\alpha} x_{1}}{s^{\alpha+1}} \geq \frac{c \alpha^{2} S^{\alpha} x_{1}}{4 s^{\alpha+1}} \geq \frac{1}{8} c \alpha^{2} x_{1}
$$

if $\alpha \geq 4 b$.

(3)

$$
\begin{aligned}
I_{3}:= & \left(\frac{2 \alpha}{s}-H\right) \frac{a^{11}(x, t) c^{2}\left(S^{\alpha}-s^{\alpha}\right)^{2}}{s^{2 \alpha}}+\frac{2 a^{11} \alpha\left(S^{\alpha}-s^{\alpha}\right)}{s^{\alpha+1}}- \\
& -\partial_{t} a^{11}(x, t) \frac{c^{2}\left(S^{\alpha}-s^{\alpha}\right)^{2}}{s^{2 \alpha}} \geq \frac{\lambda \alpha c^{2}\left(S^{\alpha}-s^{\alpha}\right)^{2}}{s^{2 \alpha}}
\end{aligned}
$$

again if $\alpha \geq 4 b$. 
(4)

$$
\begin{aligned}
I_{4}:= & -a^{1 j}(x, t) \frac{\lambda c x_{j}}{2}\left(-\frac{(\alpha+1) S^{\alpha}}{s^{\alpha+2}}+\frac{1}{s^{2}}+\frac{H\left(S^{\alpha}-s^{\alpha}\right)}{s^{\alpha+1}}\right) \\
& -\partial_{t} a^{1 j}(x, t) \frac{\lambda c x_{j}}{2} \frac{S^{\alpha}-s^{\alpha}}{s^{\alpha+1}} .
\end{aligned}
$$

We are assuming $a_{\infty}^{1 j}=0,\left|a^{1 j}(x, t)\right| \lesssim\langle x\rangle^{-\epsilon}$; hence

$$
\left|I_{4}\right| \leq 2^{\alpha} \alpha c\left|x^{\prime}\right|\langle x\rangle^{-\epsilon}+\left|\partial_{t} a^{1 j}(x, t)\right| \frac{\lambda c\left|x^{\prime}\right|}{2} \frac{S^{\alpha}-s^{\alpha}}{s^{\alpha+1}} .
$$

Recall that $c \leq R^{1+\epsilon / 8} \leq x_{1}^{1+\epsilon / 8}$; hence the first term is bounded by $\frac{1}{4}\left(I_{1}+I_{2}\right)$. Also, by dilation, we can assume $\left|\partial_{t} a^{i j}\right| \leq C \ll 1$, so that the second term is bounded by $\frac{1}{4}\left(I_{1}+I_{3}\right)$. Thus,

$$
\left|I_{4}\right| \leq \frac{I_{1}}{2}+\frac{I_{2}+I_{3}}{4} \text {. }
$$

From these estimates, we obtain

$$
\left(H+\partial_{t}\right) F \geq \frac{I_{1}+I_{2}}{2} \geq \frac{1}{32}\left(b\left|x^{\prime}\right|^{2}+c \alpha^{2} x_{1}\right) .
$$

As an approximation of $F$, we choose

$$
\begin{aligned}
F_{0}(x, t)= & \frac{\lambda}{16 s^{2}} \sum_{i, j \neq 1}\left(2 \delta_{i j}-\lambda a^{i j}(X, t)\right) x_{i} x_{j}-\frac{c \alpha S^{\alpha} x_{1}}{s^{\alpha+1}} \\
& -a^{11}(X, t) \frac{c^{2}\left(S^{\alpha}-s^{\alpha}\right)^{2}}{s^{2 \alpha}}-\sum_{j \neq 1} a^{1 j}(X, t) \frac{\lambda x_{j}}{2 s} \frac{c\left(S^{\alpha}-s^{\alpha}\right)}{s^{\alpha}},
\end{aligned}
$$

where $X=(R, 0, \ldots, 0)$. A simple calculation shows that

$$
\left|\Delta F_{0}\right| \lesssim\langle x\rangle^{-1-\epsilon}\left(\left|x^{\prime}\right|+c\right)+1 \lesssim 1
$$

and

$$
\begin{aligned}
\left|\left\langle A \nabla\left(F-F_{0}\right), \nabla \log G\right\rangle\right| & \lesssim\langle x\rangle^{-1-\epsilon}\left(\left|x^{\prime}\right|+c\right)^{3}+R^{-\epsilon}\left(\left|x^{\prime}\right|+c\right)^{2} \\
& \lesssim R^{-\epsilon / 2}\left(b\left|x^{\prime}\right|^{2}+c \alpha^{2} x_{1}\right)
\end{aligned}
$$

(the implicit constants depend on $\lambda$ but not on $R$ ).

It follows that for large $R$,

$$
M_{0}=\left(\partial_{t}+H\right) F+\Delta F_{0}-\left\langle A \nabla\left(F-F_{0}\right), \nabla \log G\right\rangle \gtrsim b\left|x^{\prime}\right|^{2}+c \alpha^{2} x_{1} .
$$

To control the remaining term, we use the Cauchy-Schwarz inequality

$$
\left|\int_{\mathbb{R}_{+}^{n+1}} u\left\langle A \nabla u, \nabla\left(F-F_{0}\right)\right\rangle G d x d t\right| \leq \frac{1}{4} \int u^{2} M_{0} G d x d t+\frac{b}{4} \int|\nabla u|^{2} G d x d t .
$$

As $|F| \lesssim\left|x^{\prime}\right|^{2}+R^{2+\epsilon}$, the lemma is proved.

\section{ACKNOWLEDGEMENT}

The author would like to thank his advisor, Carlos Kenig, for suggesting the problem, and for his invaluable guidance and support. 


\section{REFERENCES}

1. Giovanni Alessandrini and Sergio Vessella, Remark on the strong unique continuation property for parabolic operators, Proc. Amer. Math. Soc. 132 (2004), no. 2, 499-501 (electronic). MR 2022375 (2004j:35034)

2. L. Escauriaza, F. J. Fernández, and S. Vessella, Doubling properties of caloric functions, Appl. Anal. 85 (2006), no. 1-3, 205-223. MR2198840 (2006k:35121)

3. L. Escauriaza, C. E. Kenig, G. Ponce, and L. Vega, Decay at infinity of caloric functions within characteristic hyperplanes, Math. Res. Lett. 13 (2006), no. 2-3, 441-453. MR2231129

4. - On uniqueness properties of solutions of Schrödinger equations, Comm. Partial Differential Equations 31 (2006), no. 10-12, 1811-1823. MR.2273975

5. L. Escauriaza, G. Seregin, and V. Šverák, Backward uniqueness for parabolic equations, Arch. Ration. Mech. Anal. 169 (2003), no. 2, 147-157. MR2005639 (2005j:35097)

6. _ Backward uniqueness for the heat operator in half-space, Algebra i Analiz 15 (2003), no. 1, 201-214. MR1979722 (2004c:35153)

7. Luis Escauriaza and Francisco Javier Fernández, Unique continuation for parabolic operators, Ark. Mat. 41 (2003), no. 1, 35-60. MR1971939 (2004b:35136)

8. F. J. Fernandez, Unique continuation for parabolic operators. II, Comm. Partial Differential Equations 28 (2003), no. 9-10, 1597-1604. MR2001174 (2004i:35161)

9. C. E. Kenig, Personal communication.

10. O.A. Ladyzhenskaya, V.A. Solonnikov, and N.N. Uralceva, Linear and quasilinear equations of parabolic type, Translations of Mathematical Monographs, vol. 23, Amer. Math. Soc., Providence, RI, 1967. MR0241822(39:3159b)

11. E. M. Landis and O. A. Oleĭnik, Generalized analyticity and certain properties, of solutions of elliptic and parabolic equations, that are connected with it, Uspehi Mat. Nauk 29 (1974), no. 2 (176), 190-206, Collection of articles dedicated to the memory of Ivan Georgievič Petrovskir(1901-1973), I. MR0402268 (53:6089)

Department of Mathematics, University of Chicago, 5734 S. University Ave., Chicago, ILLINOIS 60637

E-mail address: tu@math.uchicago.edu

Current address: Department of Mathematics, University of Washington, Box 354350, Seattle, Washington 98195-4350

E-mail address: anhtu@math.washington.edu 